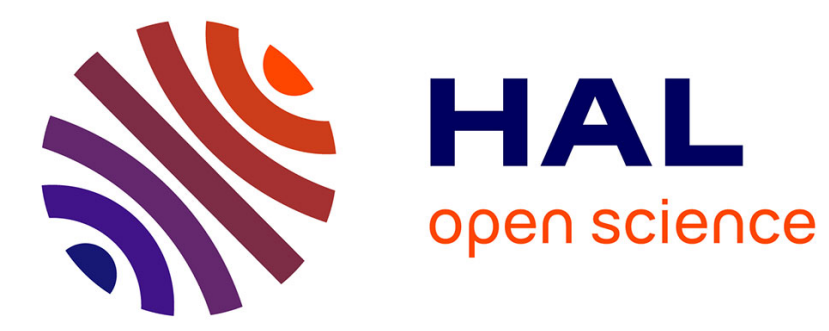

\title{
Periodic homogenization for convex functionals using Mosco convergence
}

\author{
Alain Damlamian, Nicolas Meunier, Jean van Schaftingen
}

\section{To cite this version:}

Alain Damlamian, Nicolas Meunier, Jean van Schaftingen. Periodic homogenization for convex functionals using Mosco convergence. Ricerche di matematica, 2008, 57 (2), pp.209-249. hal-00267473

\section{HAL Id: hal-00267473 \\ https://hal.science/hal-00267473}

Submitted on 27 Mar 2008

HAL is a multi-disciplinary open access archive for the deposit and dissemination of scientific research documents, whether they are published or not. The documents may come from teaching and research institutions in France or abroad, or from public or private research centers.
L'archive ouverte pluridisciplinaire HAL, est destinée au dépôt et à la diffusion de documents scientifiques de niveau recherche, publiés ou non, émanant des établissements d'enseignement et de recherche français ou étrangers, des laboratoires publics ou privés. 


\title{
Periodic homogenization for convex functionals using Mosco convergence
}

\author{
Alain Damlamian, Nicolas Meunier ${ }^{\dagger}$ and Jean Van Schaftingen ${ }^{\ddagger}$
}

27 mars 2008

\begin{abstract}
Résumé
Nous étudions les liens entre la convergence de Mosco pour des suites de fonctions convexes propres semi-continues inférieurement définies sur un espace de Banach réflexif et la convergence des suites des sousdifférentiels vus comme opérateurs maximaux monotones. Nous appliquons ces résultats pour étudier l'homogénéisation par la méthode de l'éclatement (voir [10]) des équations de la forme $-\operatorname{div} d_{\varepsilon}=f$, avec $\left(\nabla u_{\varepsilon}(x), d_{\varepsilon}(x)\right) \in \partial \varphi_{\varepsilon}(x,$.$) et où \varphi_{\varepsilon}(x,$.$) est une fonction convexe de$ Carathéodory satisfaisant des conditions de croissance et de coercivité appropriées.
\end{abstract}

\begin{abstract}
We study the relationship between the Mosco convergence of a sequence of convex proper lower semicontinuous functionals, defined on a reflexive Banach space, and the convergence of their subdifferentiels as maximal monotone graphs. We then apply these results together with the unfolding method (see [10]) to study the homogenization of equations of the form $-\operatorname{div} d_{\varepsilon}=f$, with $\left(\nabla u_{\varepsilon}(x), d_{\varepsilon}(x)\right) \in \partial \varphi_{\varepsilon}(x)$ where $\varphi_{\varepsilon}(x,$.$) is a Carathéodory convex function with suitable growth$ and coercivity conditions.
\end{abstract}

\section{Introduction}

In this paper, we consider the homogenization of the problem

$$
\begin{cases}-\operatorname{div} d_{\varepsilon}=f & \text { in } \Omega, \\ \left(\nabla u_{\varepsilon}(x), d_{\varepsilon}(x)\right) \in \partial \varphi_{\varepsilon}(x) & \text { in } \Omega, \\ u_{\varepsilon}=0 & \text { on } \partial \Omega,\end{cases}
$$

${ }^{*}$ Université Paris-Est, Laboratoire d'Analyse et de Mathématiques Appliquées CNRS UMR 8050, 94010 Créteil Cedex, France, damlamian@univ-paris12.fr

${ }^{\dagger}$ Université Paris Descartes, MAP5, 45-47 Rue des Saints Pères 75006 Paris, France, Nicolas.Meunier@math-info.univ-paris5.fr

¥Université catholique de Louvain, Département de Mathématique, Chemin du Cyclotron 2, 1348 Louvain-la-Neuve, Belgique, Jean.VanSchaftingen@uclouvain.be 
where $\varphi_{\varepsilon}: \Omega \times \mathbf{R}^{N} \rightarrow \mathbf{R}$ is Carathéodory and $\varphi_{\varepsilon}(x, \cdot)$ is convex. The subdifferential of $\varphi_{\varepsilon}(x, \cdot)$ is a multivalued map whose graph is $\partial \varphi_{\varepsilon}(x) \subset$ $\mathbf{R}^{N} \times \mathbf{R}^{N}$. The solutions of this problem are the minimizers of the functional

$$
u \mapsto \int_{\Omega} \varphi_{\varepsilon}(x, \nabla u(x)) \mathrm{d} x-\int_{\Omega} f(x) u(x) \mathrm{d} x .
$$

Thus, under suitable growth and coerciveness assumptions on $\varphi_{\varepsilon}$, this problem has at least one solution. This solution need not be unique. The homogenization problem has been addressed since the late 1970's via the theory of $\Gamma$-convergence.

In a previous paper [14], we considered the homogenization of

$$
\left\{\begin{array}{l}
-\operatorname{div} d_{\varepsilon}=f \text { in } \Omega, \\
\left(\nabla u_{\varepsilon}(x), d_{\varepsilon}(x)\right) \in A_{\varepsilon}(x),
\end{array}\right.
$$

where $A_{\varepsilon}: \Omega \rightarrow \mathfrak{M}\left(\mathbf{R}^{N} \times \mathbf{R}^{N}\right)$ is a measurable map taking its value in the set $\mathfrak{M}\left(\mathbf{R}^{N} \times \mathbf{R}^{N}\right)$ of maximal monotone graphs from $\mathbf{R}^{N}$ to $\mathbf{R}^{N}$. Under suitable growth and coerciveness assumptions, this problem has a least one solution which need not to be unique [8, 17]. There are many papers in the litterature on the study of $G$-convergence and homogenization which concern the non-linear case. We only refer to [9] and the bibliography therein.

The application of the unfolding method to problem (1.2) lead us to the topic of convergence of maximal monotone operators. This subject was actively developed in the 1970's, most particularly in the Hilbert space setting [1, 2, 国. It had been studied at the time by the first author in the case of reflexive Banach space and lead to a paper that was never submitted for publication [12].

In [14, particular attention was given to the relationship between pointwise and global convergence of graphs. More precisely, if $A, A_{n}: \tilde{\Omega} \rightarrow$ $\mathfrak{M}\left(X \times X^{\prime}\right)$ are measurable maps whose values are maximal monotone operators, one can define the operators

$$
\mathcal{A}_{n}=\left\{(u, v) \in L^{p}(\tilde{\Omega} ; X) \times L^{q}\left(\tilde{\Omega} ; X^{\prime}\right) \mid(u(t), v(t)) \in A_{n}(t) \text { for a.e. } t \in \tilde{\Omega}\right\}
$$

and $\mathcal{A}$ similarly. We looked for conditions under which the convergence $A_{n}(t) \longmapsto A(t)$ for almost every $t \in \Omega$ implies $\mathcal{A}_{n} \longmapsto \mathcal{A}$. Since subdifferentials of lower semicontinuous convex functions are maximal monotone graphs, problem (1.1) is a special case of problem (1.2). The convergence of the subdifferentials (in the sense of graphs) is equivalent to the Mosco convergence of the associated (normalized) convex functionals. This case was treated in the reflexive Banach space setting in the unpublished paper [12]. If $\varphi, \varphi_{n}: \Omega \times X \rightarrow \mathbf{R} \cup\{+\infty\}$ are normal convex integrands, define

$$
I_{\varphi}(u)=\int_{\Omega} \varphi(x, u(x)) \mathrm{d} x
$$


and $I_{\varphi_{n}}$ similarly. In Theorem 3.12, we give sufficient conditions for the Mosco convergence $\varphi_{n}(t,.) \stackrel{\mathcal{M}}{\longrightarrow} \varphi(t,$.$) to implie that of I_{\varphi_{n}} \stackrel{\mathcal{M}}{\longrightarrow} I_{\varphi}$ and thus the convergence of the induced graphs $\mathcal{A}_{\varphi_{n}}$, where

$\mathcal{A}_{\varphi_{n}}=\left\{(u, v) \in L^{p}(\Omega ; X) \times L^{q}\left(\Omega ; X^{\prime}\right) \mid(u(t), v(t)) \in \partial \varphi_{n}(t)\right.$ for a.e. $\left.t \in \Omega\right\}$.

Then, (1.1) can be treated using Mosco convergence in the general setting of a normal convex integrand. Interestingly enough, the obtained result differs from the one which follows the application of the theory of $\Gamma$-convergence, classically used with the strong topology on a space of type $L^{p}$ together with a coerciveness hypothesis (thus implying that the effective domains of the functions remain included in the corresponding $W^{1, p}$ space).

The outline of the paper is as follows. Section 2 recalls the definition of maximal monotone operators, subdifferentials, the convergence of maximal monotone graphs and the Mosco convergence. In section 3, using [12], we consider the integration of normal convex integrands and we prove key results about the Mosco convergence of functions and the Mosco convergence of the associated integral functional. In section 4 we recall the definition of the unfolding operator, averaging operator and the corresponding convergence properties (cf. [10], [13]). The statements can also be found in 19] and [20]. Finally, we consider the homogenization problem in section 5 .

Some of the results of this paper were announced in [20] and [14].

\section{The subdifferentials of Convex functions as max- imal monotone graphs}

In this section we recall basic facts about convex functions, their subdifferentials and we examine the relationship between the convergence of convex functions and convergence of their subdifferentials.

\subsection{Subdifferentials of convex functions}

We shall work in the framework of a reflexive Banach space $X$ whose dual is $X^{\prime}$. The norms on $X$ and $X^{\prime}$ are denoted by $\|\cdot\|_{X},\|\cdot\|_{X^{\prime}}$ respectively (or $\|\cdot\|$ when no confusion arises), and the duality pairing of $X^{\prime}$ and $X$ by $\langle\cdot, \cdot\rangle$. Since $X$ is reflexive, there is an equivalent norm on $X$ which is locally uniformly convex as well as its dual [27]. We shall therefore assume that such a norm is used from now on, so that $X$ and $X^{\prime}$ are locally uniformly convex. Let $X$ be a Banach space. Its norm is locally uniformly convex whenever the following holds:

$\forall \xi \in X, \forall \varepsilon>0, \exists \delta>0, \forall \zeta \in X,\left\|\frac{\xi+\zeta}{2}\right\|>\max (\|\xi\|,\|\zeta\|)-\delta \Rightarrow\|x-y\|<\varepsilon$ 
The main property of such a locally uniformly convex norm is the following well-known result:

Proposition 2.1. Let $X$ be a Banach space with a locally uniformly convex norm. Then the strong convergence in $X$ is equivalent to the weak convergence together with the convergence of the norms:

$$
\xi_{n} \rightarrow \xi \Longleftrightarrow \xi_{n} \rightarrow \xi \text { and }\left\|\xi_{n}\right\| \rightarrow\|\xi\| .
$$

This is the only property, consequence of local uniform convexity, which will be used both in $X$ and in $X^{\prime}$. It is of course satisfied in the case of uniformly convex spaces, and in particular for $L^{p}$ spaces for $1<p<\infty$.

The duality mapping $F: X \rightarrow X^{\prime}$ maps each $\xi \in X$ to $F(\xi) \in X^{\prime}$ such that $\|F(\xi)\|_{X^{\prime}}=\|\xi\|_{X}$ and $\langle F(\xi), \xi\rangle=\|\xi\|_{X}^{2}$. By local uniform convexity, $F$ is single-valued and monotone as well as its inverse (which is the duality mapping from $X^{\prime}$ to $X$ ) and both are homeomorphism.

We can now turn to convex functions defined on $X$. A function $\varphi: X \rightarrow$ $\mathbf{R} \cup\{+\infty\}$ will be said to be proper if $\varphi(X) \neq\{+\infty\}$. Its epigraph is

$$
\text { epi } \varphi=\{(\xi, \tau) \in X \times \mathbf{R} \cup\{+\infty\}: \varphi(\xi) \leq \tau\}
$$

The epigraph is a non empty closed convex subset if and only if $\varphi$ is a proper lower semicontinuous convex function. The effective domain of $\varphi$ is defined by

$$
D(\varphi)=\{\xi \in X: \varphi(\xi) \in \mathbf{R}\} .
$$

Definition 2.2. The subdifferential $\partial \varphi$ at $\xi \in D(\varphi)$ of the function $\varphi$ : $X \rightarrow \mathbf{R} \cup\{+\infty\}$ is the set

$$
\partial \varphi(\xi)=\left\{\eta \in X^{\prime}: \forall \zeta \in X, \varphi(\zeta) \geq \varphi(\xi)+\langle\eta, \zeta-\xi\rangle\right\} .
$$

Remark 1. The definition of subdifferential does not require that the function $\varphi$ be convex, only that it be proper. It is worth to note that, in this generality, $\varphi$ attains its minimum at $\xi \in X$ if and only if $0 \in \partial \varphi(\xi)$.

Definition 2.3. For $\lambda>0$ and $\zeta \in X$, define $\varphi_{\lambda}$ as the following infconvolution of the two functions $\varphi$ and $\frac{\|\cdot\|^{2}}{2 \lambda}$ :

$$
\varphi_{\lambda}(\zeta)=\inf _{\xi \in X} \varphi(\xi)+\frac{\|\zeta-\xi\|^{2}}{2 \lambda} .
$$

Lemma 2.4. Let $\varphi: X \rightarrow \mathbf{R} \cup\{+\infty\}$ be a lower semicontinuous proper convex function. For every $\lambda>0, \varphi_{\lambda} \in C^{1}(X, \mathbf{R})$, and for every $\xi \in X$, there exists a unique $\xi_{\lambda} \in X$ such that

$$
\varphi_{\lambda}(\xi)=\varphi\left(\xi_{\lambda}\right)+\frac{\left\|\xi-\xi_{\lambda}\right\|^{2}}{2 \lambda},
$$


or equivalently

$$
\lambda^{-1} F\left(\xi-\xi_{\lambda}\right) \in \partial \varphi\left(\xi_{\lambda}\right) \text { i.e. } \xi=\xi_{\lambda}+F^{-1}\left(\lambda \eta_{\lambda}\right) \text { with } \eta_{\lambda} \in \partial \varphi\left(\xi_{\lambda}\right) .
$$

Moreover, for every $\xi$ in $D(\varphi), \xi_{\lambda} \rightarrow \xi$ as $\lambda \rightarrow 0$ and

$$
\lim _{\lambda \rightarrow 0} \varphi_{\lambda}(\xi)=\lim _{\lambda \rightarrow 0} \varphi\left(\xi_{\lambda}\right)=\varphi(\xi) .
$$

Definition 2.5. Following the standard notations of maximal monotone operator theory, for every $\xi \in X$, the vector $\xi_{\lambda}$ from the Lemma 2.4 is denoted $J_{\lambda}^{\partial \varphi}(\xi)$ and the map $J_{\lambda}^{\partial \varphi}$ is called the $(\lambda-)$ resolvant for $\partial \varphi$. Similarly, $\eta_{\lambda}$ is denoted $\partial \varphi_{\lambda}(\xi)$, and the map $\partial \varphi_{\lambda}$ is the Yosida approximation of $\partial \varphi$.

The proof of the previous Lemma is a classical generalization of the same result in the Hilbert case. For the convenience of the reader we recall it here.

Proof of Lemma 2.4. For fixed $\xi \in X$ and $\lambda>0$, the strict convexity of the norm implies the uniqueneness of $\xi_{\lambda}$. To prove the existence of $\xi_{\lambda}$, let $\xi_{\lambda, n}$ be a minimizing sequence for $\zeta \mapsto \varphi(\zeta)+\frac{\|\xi-\zeta\|^{2}}{2 \lambda}$, i.e.

$$
\varphi\left(\xi_{\lambda, n}\right)+\frac{\left\|\xi-\xi_{\lambda, n}\right\|^{2}}{2 \lambda} \rightarrow \varphi_{\lambda}(\xi) \text { as } n \rightarrow+\infty .
$$

Since $\varphi$ is lower semi-continuous convex and proper, by Hahn-Banach's theorem, it is bounded below on $X$ by a continuous affine function, hence $\varphi_{\lambda}(\xi)$ is finite for every $\xi \in X$ and $\lambda>0$. By the same argument,

$$
\frac{\left\|\xi-\xi_{\lambda, n}\right\|^{2}}{2 \lambda} \leq \varphi_{\lambda}(\xi)+a\left\|\xi_{\lambda, n}\right\|+b
$$

so the sequence $\left(\xi_{\lambda, n}\right)_{n \geq 1}$ is bounded. One can extract a weakly convergent subsequence : $\xi_{\lambda, n_{k}} \rightarrow \xi_{\lambda}$. By weak lower semi-continuity of the norm and of $\varphi, \liminf _{k \rightarrow \infty}\left\|\xi-\xi_{\lambda, n_{k}}\right\| \geq\left\|\xi-\xi_{\lambda}\right\|$ and $\liminf _{k \rightarrow \infty} \varphi\left(\xi_{\lambda, n_{k}}\right) \geq \varphi\left(\xi_{\lambda}\right)$. However, by the previous convergence, $\varphi\left(\xi_{\lambda, n}\right)+\frac{1}{2 \lambda}\left\|\xi-\xi_{\lambda, n}\right\|^{2}$ converges to $\varphi_{\lambda}(\xi)$ which is bounded above by $\varphi\left(\xi_{\lambda}\right)+\frac{1}{2 \lambda}\left\|\xi-\xi_{\lambda}\right\|^{2}$. As a consequence, $\lim _{k \rightarrow \infty} \varphi\left(\xi_{\lambda, n_{k}}\right)=\varphi\left(\xi_{\lambda}\right), \lim _{k \rightarrow \infty}\left\|\xi-\xi_{\lambda, n_{k}}\right\|=\left\|\xi-\xi_{\lambda}\right\|$ and $\varphi_{\lambda}(\xi)=\varphi\left(\xi_{\lambda}\right)+$ $\frac{1}{2 \lambda}\left\|\xi-\xi_{\lambda}\right\|^{2}$. This prove the existence of $\xi_{\lambda}$.

Since $\varphi_{\lambda}(\xi) \leq \varphi(\xi), \lim \sup _{\lambda \rightarrow 0} \varphi_{\lambda}(\xi) \leq \varphi(\xi)$. Moreover, due to

$$
\frac{\left\|\xi_{\lambda}-\xi\right\|^{2}}{2 \lambda}=\varphi_{\lambda}(\xi)-\varphi\left(\xi_{\lambda}\right) \leq \varphi_{\lambda}(\xi)+a\left\|\xi_{\lambda}\right\|+b,
$$

one has $\xi_{\lambda} \rightarrow \xi$ as $\lambda \rightarrow 0$. Finally, by lower semicontinuity, we deduce that

$$
\varphi(\xi) \leq \liminf _{\lambda \rightarrow 0} \varphi\left(\xi_{\lambda}\right)+\frac{\left\|\xi-\xi_{\lambda}\right\|^{2}}{2 \lambda}=\liminf _{\lambda \rightarrow 0} \varphi_{\lambda}(\xi) .
$$


Definition 2.6. For $p \geq 1$, one can also define the inf-convolution $\varphi_{\lambda, p}$ of $\varphi$ with $\frac{1}{\lambda^{p-1}} \frac{\|\cdot\|^{p}}{p}$ :

$$
\varphi_{\lambda, p}(\xi)=\inf _{\zeta \in X} \varphi(\zeta)+\frac{1}{\lambda^{p-1}} \frac{\|\zeta-\xi\|^{p}}{p},
$$

which is often better suited (in particular for $L^{p}$-type spaces, as in section 5).

For $p>1$, reasoning as in Lemma 2.4, one can show that $\varphi_{\lambda, p}$ belongs to $C^{1}(X ; \mathbf{R})$ and that $\lim _{\lambda \rightarrow 0} \varphi_{\lambda, p}(\xi) \rightarrow \varphi(\xi)$, 12]. The case $p=1$ is more complicated and we refer to [3] for details.

Proposition 2.7. For given $\varphi$ lower semicontinuous convex and proper on $X$, for every $\lambda>0$, the maps $J_{\lambda}^{\partial \varphi}$ and $\partial \varphi_{\lambda}$ are continuous from $X$ to $X$ and $X^{\prime}$ respectively. Furthermore, $\partial \varphi_{\lambda}$ is actually the Fréchet derivative (as well as the subdifferential) of $\varphi_{\lambda}$ (which is why there is no need for parentheses in the notation $\partial \varphi_{\lambda}$ ).

The notations $J_{\lambda, p}^{\partial \varphi}$ and $\partial \varphi_{\lambda, p}$ are used for $p \geq 1, p \neq 2$ with similar properties.

Proof. We give the proof for $p=2$ (the case $p \neq 2$ is similar).

Consider a sequence $\xi_{n} \rightarrow \xi$, and use the notations $\xi_{\lambda} \doteq J_{\lambda}^{\partial \varphi}(\xi),\left(\xi_{n}\right)_{\lambda} \doteq$ $J_{\lambda}^{\partial \varphi}\left(x_{n}\right)$. First,

$$
\varphi_{\lambda}(\xi)=\varphi\left(\xi_{\lambda}\right)+\frac{\left\|\xi-\xi_{\lambda}\right\|^{2}}{2 \lambda}=\lim _{n \rightarrow \infty}\left(\varphi\left(\xi_{\lambda}\right)+\frac{\left\|\xi_{n}-\xi_{\lambda}\right\|^{2}}{2 \lambda}\right) \geq \limsup _{n \rightarrow \infty} \varphi_{\lambda}\left(\xi_{n}\right) .
$$

Moreover, by (2.2),

$$
\frac{\left\|\xi_{n}-\left(\xi_{n}\right)_{\lambda}\right\|^{2}}{2 \lambda} \leq \varphi_{\lambda}\left(\xi_{n}\right)+a\left\|\left(\xi_{n}\right)_{\lambda}\right\|+b
$$

hence the sequence $\left(\left(\xi_{n}\right)_{\lambda}\right)_{n \geq 1}$ is bounded. Taking a subsequence $\left(\left(\xi_{n_{k}}\right)_{\lambda}\right)_{k \geq 1}$ such that $\left(\xi_{n_{k}}\right)_{\lambda} \rightarrow \xi_{0}$, and making use of both $\liminf _{n \rightarrow \infty} \varphi\left(\left(\xi_{n}\right)_{\lambda}\right) \geq \varphi\left(\xi_{0}\right)$ and $\liminf _{n \rightarrow \infty}\left\|\xi_{n}-\left(\xi_{n}\right)_{\lambda}\right\| \geq\left\|\xi-\xi_{0}\right\|$ one obtains

$$
\begin{aligned}
\liminf _{n \rightarrow+\infty} \varphi_{\lambda}\left(\xi_{n}\right) & =\liminf _{n \rightarrow+\infty}\left(\varphi\left(\left(\xi_{n}\right)_{\lambda}\right)+\frac{\left\|\left(\xi_{n}\right)_{\lambda}-\xi_{n}\right\|^{2}}{2 \lambda}\right) \\
& \geq \varphi\left(\xi_{0}\right)+\frac{\left\|\xi_{0}-\xi\right\|^{2}}{2 \lambda} \\
& \geq \varphi_{\lambda}(\xi)
\end{aligned}
$$

so that, recalling (2.3), $\lim _{n \rightarrow \infty} \varphi_{\lambda}\left(\xi_{n}\right)=\varphi_{\lambda}(\xi)$. Therefore, the function $\varphi_{\lambda}$ is continuous. Furthermore, from the previous equality, it follows

$$
\lim _{n \rightarrow \infty} \varphi\left(\left(\xi_{n}\right)_{\lambda}\right)=\varphi\left(\xi_{0}\right) \text { and } \lim _{n \rightarrow \infty} \frac{\left\|\left(\xi_{n}\right)_{\lambda}-\xi_{n}\right\|^{2}}{2 \lambda}=\frac{\left\|\xi_{0}-\xi\right\|^{2}}{2 \lambda} .
$$


Again, by local uniform convexity of the norm, one concludes that $\left(\xi_{n}\right)_{\lambda}$ converges strongly to $\xi_{\lambda}$. So $x \mapsto J_{\lambda}^{\partial \varphi}(\xi)$ is continuous. By the continuity of the duality map, so is $\xi \mapsto \partial \varphi_{\lambda}(\xi)$.

Let us now show that $\partial \varphi_{\lambda}$ is the Fréchet derivative of $\varphi_{\lambda}$. By definition,

$$
\lambda^{-1} F\left(\xi-\xi_{\lambda}\right) \in \partial \varphi\left(\xi_{\lambda}\right) .
$$

For every $\zeta \in X$ denote $J_{\lambda}^{\partial \varphi}(\zeta)$ by $\zeta_{\lambda}$. By definition of the subdifferential, $\varphi\left(\xi_{\lambda}\right)+\lambda^{-1}\left\langle F\left(\xi-\xi_{\lambda}\right), \zeta_{\lambda}-\xi_{\lambda}\right\rangle \leq \varphi\left(\zeta_{\lambda}\right)$ so that

$$
\begin{aligned}
\varphi_{\lambda}(\xi) & =\varphi\left(\xi_{\lambda}\right)+\frac{\left\|\xi-\xi_{\lambda}\right\|^{2}}{2 \lambda} \\
& \leq \varphi\left(\zeta_{\lambda}\right)-\lambda^{-1}\left\langle F\left(\xi-\xi_{\lambda}\right), \zeta_{\lambda}-\xi_{\lambda}\right\rangle+\frac{\left\|\xi-\xi_{\lambda}\right\|^{2}}{2 \lambda} \\
& =\varphi_{\lambda}(\zeta)+\lambda^{-1}\left(\left\langle F\left(\xi-\xi_{\lambda}\right), \xi_{\lambda}-\zeta_{\lambda}\right\rangle+\frac{\left\|\xi-\xi_{\lambda}\right\|^{2}}{2}-\frac{\left\|\zeta-\zeta_{\lambda}\right\|^{2}}{2}\right) \\
& \leq \varphi_{\lambda}(\zeta)+\lambda^{-1}\left\langle F\left(\xi-\xi_{\lambda}\right), \xi-y\right\rangle,
\end{aligned}
$$

since

$$
\frac{\left\|\xi-\xi_{\lambda}\right\|^{2}}{2}+\left\langle F\left(\xi-\xi_{\lambda}\right), \zeta-\xi+\xi_{\lambda}-\zeta_{\lambda}\right\rangle \leq \frac{\left\|\zeta-\zeta_{\lambda}\right\|^{2}}{2} .
$$

It follows that

$$
\varphi_{\lambda}(\xi)+\lambda^{-1}\left\langle F\left(\xi-\xi_{\lambda}\right), \zeta-\xi\right\rangle \leq \varphi_{\lambda}(\zeta) .
$$

Exchanging the roles of $x$ and $y$, this gives

$\varphi_{\lambda}(\zeta) \leq \varphi_{\lambda}(\xi)+\lambda^{-1}\left\langle F\left(\xi-\xi_{\lambda}\right), \zeta-\xi\right\rangle+\lambda^{-1}\left\langle F\left(\zeta-\zeta_{\lambda}\right)-F\left(\xi-\xi_{\lambda}\right), \zeta-\xi\right\rangle$.

Since $F$ is continuous and $\zeta_{\lambda} \rightarrow \xi_{\lambda}$ as $\zeta \rightarrow \xi$, it follows from (2.4) and (2.5) that $\varphi_{\lambda}$ is Fréchet differentiable at $\xi$ and its derivative is $\lambda^{-1} F\left(\xi-\xi_{\lambda}\right)=$ $\partial \varphi_{\lambda}(\xi)$.

The connection between the Yosida approximations for different values of the parameter $\lambda$ is given by the next statements.

Lemma 2.8. Let $\alpha$ and $\beta$ be given in $X, \lambda$ and $\mu$ positive, then

$$
\inf _{\xi \in X}\left(\frac{1}{2 \lambda}\|\beta-\xi\|^{2}+\frac{1}{2 \mu}\|\xi-\alpha\|^{2}\right)=\frac{1}{2(\lambda+\mu)}\|\beta-\alpha\|^{2} .
$$

Similarly, for $p \geq 1$,

$$
\inf _{\xi \in X}\left(\frac{1}{p \lambda^{p-1}}\|\beta-\xi\|^{p}+\frac{1}{p \mu^{p-1}}\|\xi-\alpha\|^{p}\right)=\frac{1}{p(\lambda+\mu)^{p-1}}\|\beta-\alpha\|^{p} .
$$


Proof. The function $\|\cdot\|$ being Fréchet differentiable and strictly convex, the infimum of the left-hand side is achieved at a unique point $\xi$ such that $\frac{1}{\lambda} F(\beta-\xi)=\frac{1}{\mu} F(\xi-\alpha)\left(\right.$ resp. $\left.\frac{1}{\lambda^{p-1}} F_{p}(\beta-\xi)=\frac{1}{\mu^{p-1}} F_{p}(\xi-\alpha)\right)$. We deduce that $\frac{1}{\lambda}(\beta-\xi)=\frac{1}{\mu}(\xi-\alpha)$, so $\xi-\beta=\frac{\lambda}{\lambda+\mu}(\alpha-\beta)$ and $\xi-\alpha=\frac{\mu}{\lambda+\mu}(\beta-\alpha)$, from which the conclusions follow.

As a direct consequence, we get

Proposition 2.9. Let $\varphi$ be lower semicontinuous convex and proper on $X$ and $\lambda$ and $\mu$ be positive. Let $\psi$ denote the Yosida approximation $\varphi_{\lambda}$ of $\varphi$. Then, the Yosida approximation $\psi_{\mu}$ of $\psi$ is $\varphi_{(\lambda+\mu)}:\left(\varphi_{\lambda}\right)_{\mu}=\varphi_{(\lambda+\mu)}$, and the Yosida approximation $\left(\partial \varphi_{\lambda}\right)_{\mu}$ of $\partial \varphi_{\lambda}$ is just $\partial \varphi_{(\lambda+\mu)}$.

The same is true for the modified approximations: $\left(\varphi_{\lambda, p}\right)_{\mu, p}=\varphi_{(\lambda+\mu), p}$ and their subdifferentials.

Definition 2.10. Let $\varphi: X \rightarrow \mathbf{R} \cup\{+\infty\}$ be a lower semicontinuous proper convex function. The convex conjugate $\varphi^{*}: X^{\prime} \rightarrow \mathbf{R} \cup\{+\infty\}$ of $\varphi$ is defined by

$$
\varphi^{*}(\eta)=\sup _{\xi \in X}(\langle\eta, \xi\rangle-\varphi(\xi))
$$

Example 1. For $1<p<\infty$, the function $\varphi: X \rightarrow \mathbf{R}: \xi \mapsto \frac{1}{p}\|\xi\|^{p}$ is a convex continuous function. Furthermore, $\partial \varphi(\xi)=\|\xi\|^{p-2} F(\xi)$, and, for every $\eta \in X^{\prime}, \varphi^{*}(\eta)=\frac{1}{q}\|\eta\|^{q}$, where $\frac{1}{p}+\frac{1}{q}=1$.

Example 2. The following equality holds (a straightforward consequence of the fact that the conjugate of an inf-convolution is the sum of the conjugates):

$$
\left(\varphi_{\lambda, p}\right)^{*}(\eta)=\varphi^{*}(\eta)+\lambda^{p-1} \frac{\|\eta\|^{q}}{q}
$$

The next proposition is a well-known consequence of the Hahn-Banach Theorem (see for example [16]).

Proposition 2.11. Let $\varphi: X \rightarrow \mathbf{R} \cup\{+\infty\}$ be a lower semicontinuous proper convex function, then $\varphi^{*}$ is proper, lower semicontinuous and convex, $\varphi^{* *}=\varphi$ and for every $(\xi, \eta) \in X \times X^{\prime}$,

$$
\varphi(\xi)+\varphi^{*}(\eta) \geq\langle\eta, \xi\rangle \quad \text { (Young's inequality) }
$$

holds, with equality if and only if $(\xi, \eta) \in \partial \varphi$. In particular,

$$
\partial \varphi^{*}=\{(\eta, \xi):(\xi, \eta) \in \partial \varphi\} .
$$

The following theorem of Fenchel and Rockafellar gives the relationship between the conjugate of a function and that of its restriction to a closed subspace. 
Proposition 2.12 (Fenchel-Rockafellar). Let $V \subset X$ be a closed subspace, $\varphi: X \rightarrow \mathbf{R} \cup\{+\infty\}$ be a lower semicontinuous convex function such that $\varphi$ is continuous at some point $\xi \in V$. Then

$$
\inf _{\xi \in V} \varphi(\xi)=-\inf _{\eta \in V^{\perp}} \varphi^{*}(\eta)
$$

where

$$
V^{\perp}=\left\{\eta \in X^{\prime}: \forall \xi \in V,\langle\eta, \xi\rangle=0\right\}
$$

\subsection{Subdifferentials as maximal monotone operators}

The subdifferential of $\varphi$ is a set-valued operator. One can consider general set-valued operators $A: X \rightarrow X^{\prime}$, that is maps which take every point $\xi \in X$ to some subset $A \xi \subset X^{\prime}$. Traditionnaly, these applications are simply called operators and the notation $A$ is used to denote both the operator and its graph, i.e. the set $\left\{(\xi, \eta) \in X \times X^{\prime}: \eta \in A \xi\right\}$, since no confusion arises. The domain of the operator $A$ is

$$
D(A)=\{x \in X \text { such that } A x \neq \emptyset\} .
$$

An operator $A$ is monotone if for every $\left(\xi_{1}, \eta_{1}\right),\left(\xi_{2}, \eta_{2}\right) \in A$,

$$
\left\langle\eta_{1}-\eta_{2}, \xi_{1}-\xi_{2}\right\rangle \geq 0 \text {. }
$$

It is maximal monotone, if for every monotone operator $B \subset X \times X^{\prime}$ such that $A \subseteq B$, one has in fact $A=B$. For more details on maximal monotone operators see [田, 司, 6].

We now return to convex functions and their subdifferentials and recall the fundamental relationship that they have with maximal monotone operators:

Proposition 2.13 (Rockafellar [23, 25]). Let $\varphi: X \rightarrow \mathbf{R} \cup\{+\infty\}$ be a proper function. The function $\varphi$ is lower semicontinuous and convex if and only if $\partial \varphi$ is maximal monotone.

An operator $A \subset X \times X^{\prime}$ is said cyclically monotone if for any $\ell \in \mathbb{N}^{*}$ and for every $\left(\xi^{1}, \eta^{1}\right), \ldots,\left(\xi^{\ell}, \eta^{\ell}\right) \in A$ :

$$
\sum_{i=1}^{\ell}\left\langle\eta^{i}, \xi^{i+1}-\xi^{i}\right\rangle \leq 0,
$$

where by convention $\xi^{\ell+1}=\xi^{1}$.

Proposition 2.14 (Rockafellar [23, 25]). Let $A \subset X \times X^{\prime}$. The following are equivalent 
(a) there exists a lower semicontinuous proper convex function $\varphi$ such that $A=\partial \varphi$,

(b) A is cyclically monotone and maximal monotone.

Moreover, if $\varphi$ and $\psi$ are lower semicontinuous proper convex functions such that $\partial \varphi=\partial \psi$, then there exists $c \in \mathbf{R}$ such that $\psi=\varphi+c$.

The proof is based on the following abstract "integration formula" established in [23] and used therein for the construction of a $\varphi$ such that $\partial \varphi=A$ : for any $\xi$ and $\zeta$ in $X$,

$$
\begin{array}{r}
\varphi(\zeta)-\varphi(\xi)=\sup \left\{\sum_{i=1}^{\ell}\left\langle\eta^{i}, \xi^{i+1}-\xi^{i}\right\rangle: \ell \geq 1,\left(\xi^{i}, \eta^{i}\right) \in \partial \varphi\right. \\
\left.\xi^{1}=\xi \text { and } \xi^{\ell+1}=\zeta\right\} .
\end{array}
$$

\subsection{Convergence of convex functions and of maximal mono- tone graphs}

For lower semicontinuous convex functions, the Mosco convergence was introduced in [21]:

Definition 2.15. Let $\varphi_{n}$ and $\varphi$ be lower semicontinuous convex functions on $X$. The sequence $\left(\varphi_{n}\right)_{n \in \mathbf{N}}$ converges to $\varphi$ in the sense of Mosco, (denoted $\varphi_{n} \stackrel{\mathcal{M}}{\longrightarrow} \varphi$ ) whenever the following two conditions are satisfied:

(M-i) for every $\xi \in X$, there exists a sequence $\left(\xi_{n}\right)_{n \geq 1}$ in $X$ such that $\xi_{n} \rightarrow \xi$ strongly in $X$ and

$$
\limsup _{n \rightarrow+\infty} \varphi_{n}\left(\xi_{n}\right) \leq \varphi(\xi)
$$

(M-ii) for every sequence $\left(\xi_{n}\right)_{n \geq 1}$ in $X$ such that $\xi_{n} \rightarrow \xi \in X$ weakly in $X$,

Remark 2.

$$
\liminf _{n \rightarrow+\infty} \varphi_{n}\left(\xi_{n}\right) \geq \varphi(\xi) .
$$

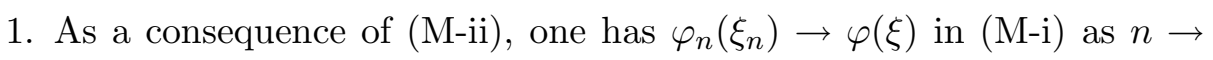
$+\infty$.

2. A constant sequence $\varphi$ Mosco-converges to itself (since a lower semicontinuous convex functions on $X$ is weakly lower semi-continuous).

3. Any subsequence of a Mosco-convergent sequence also Mosco-converges to the same limit. 
By Proposition 2.11, every lower semicontinuous proper convex function is bounded below by some continuous affine function, hence by an affine function of the norm. The following shows that the latter property is true uniformly for a sequence converging in the sense of Mosco:

Proposition 2.16 (Mosco 21]). Let $\varphi_{n}, \varphi$ be lower semicontinuous proper convex functions on $X$ such that $\varphi_{n} \stackrel{\mathcal{M}}{\longrightarrow} \varphi$, then there exists $a, b \in \mathbf{R}^{+}$such that for all $n$ and for all $\xi$ in $X$,

$$
\varphi_{n}(\xi)+a\|\xi\|+b \geq 0 .
$$

Proof. Let $\varphi_{n}$ converge to $\varphi$ in the sense of Mosco. We reason by contradiction, so we assume that, for every $k \in \mathbf{N}$, there is some $n_{k} \in \mathbf{N}$ and $\zeta_{k} \in X$ such that $\varphi_{n_{k}}\left(\zeta_{k}\right)+k\left(1+\left\|\zeta_{k}\right\|\right)<0$. Since each $\varphi_{n}$ satisfies (2.7), this implies that $n_{k} \rightarrow \infty$. Let $\xi$ be in $D(\varphi)$ and by (M-i) let $\xi_{n}$ be a sequence converging to $\xi$ with $\lim _{n \rightarrow \infty} \varphi_{n}\left(\xi_{n}\right)=\varphi(\xi)$. Set

$$
t_{k}=\min \left(1, \frac{1}{\sqrt{k}\left\|\zeta_{k}-\xi_{n_{k}}\right\|}\right) \text { and } \vartheta_{k}=t_{k} \zeta_{k}+\left(1-t_{k}\right) \xi_{n_{k}} .
$$

By convexity,

$\varphi_{n_{k}}\left(\vartheta_{k}\right) \leq t_{k} \varphi_{n_{k}}\left(\zeta_{k}\right)+\left(1-t_{k}\right) \varphi_{n_{k}}\left(\xi_{n_{k}}\right)<-k t_{k}\left(1+\left\|\zeta_{k}\right\|\right)+\left(1-t_{k}\right) \varphi_{n_{k}}\left(\xi_{n_{k}}\right)$.

As $k$ goes to $\infty$, the following hold: $t_{k} \rightarrow 0^{+}, t_{k} \zeta_{k} \rightarrow 0$, hence $\vartheta_{k} \rightarrow \xi$. By (M-ii), $\varphi(\xi) \leq \liminf _{k \rightarrow \infty} \varphi_{n_{k}}\left(\vartheta_{k}\right)$. At the limit $k \rightarrow \infty$, the previous inequality implies

$$
0 \leq \liminf _{k \rightarrow \infty}\left(-k t_{k}\left(1+\left\|\zeta_{k}\right\|\right)\right)=-\infty
$$

a contradiction.

Remark 3. If $\varphi$ is lower semicontinuous convex and is bounded below by an affine function of the norm (as in (2.7))

$$
\varphi(\xi)+a\|\xi\|+b \geq 0 \text { for all } \xi
$$

then a straightforward computation shows that for every $\lambda>0, \varphi_{\lambda}$ satisfies

$$
\varphi_{\lambda}(\xi)+a\|\xi\|+b+\frac{\lambda}{2} a^{2} \geq 0 \quad \text { for all } \xi .
$$

The converse is obvious, since $\varphi$ is bounded below by $\varphi_{\lambda}$.

Similarly, one checks that $\varphi_{\lambda, p}(\xi)+a\|\xi\|+b+\frac{\lambda}{p} a^{q} \geq 0$ for all $\xi$ (where $\left.\frac{1}{p}+\frac{1}{q}=1\right)$.

This remark applies uniformly to a sequence which Mosco-converges. 
Lemma 2.17. Let $\varphi_{n}$ and $\varphi$ be proper lower semicontinuous convex functions on $X$ such that $\varphi_{n} \stackrel{\mathcal{M}}{\longrightarrow} \varphi$ and let $U \subset X$ be open. If

$$
\sup _{n \in \mathbf{N}} \sup _{\xi \in U} \varphi_{n}(\xi)<\infty .
$$

then $\varphi_{n}$ converges uniformly to $\varphi$ on strongly compact subsets of $U$.

In particular, if the sequence $\left\{\varphi_{n}\right\}$ is locally bounded above on $X$, then its Mosco-convergence to $\varphi$ implies that it converges locally uniformly to $\varphi$ on $X$.

Proof. It suffices to prove that if $\xi_{n} \rightarrow \xi \in U$, then $\varphi_{n}\left(\xi_{n}\right) \rightarrow \varphi(\xi)$.

First, by (M-园), we have

$$
\liminf _{n \rightarrow \infty} \varphi_{n}\left(\xi_{n}\right) \geq \varphi(\xi) .
$$

To obtain the reverse inequality, start with (M-i) to exhibit a sequence $\left(\zeta_{n}\right)_{n \geq 1}$ converging strongly to $\xi$ in $X$ and such that $\varphi_{n}\left(\zeta_{n}\right) \rightarrow \varphi(\xi)$. For every $0<t<1$, convexity implies

$$
\varphi_{n}\left(\xi_{n}\right) \leq t \varphi_{n}\left(\zeta_{n}\right)+(1-t) \varphi_{n}\left(\frac{\xi_{n}-t \zeta_{n}}{1-t}\right) .
$$

By hypothesis, $\left(\zeta_{n}-t \xi_{n}\right) /(1-t)$ converges to $\xi$, so

$$
\limsup _{n \rightarrow \infty} \varphi_{n}\left(\xi_{n}\right) \leq t \varphi(\xi)+(1-t) \sup _{n \in \mathbf{N}} \sup _{\zeta \in U} \varphi_{n}(\zeta) .
$$

Letting $t \rightarrow 1^{-}$concludes the proof.

Remark 4. Proposition 2.16 together with classical properties of convex functions gives a more precise result. Under the assumptions of Lemma 2.17, the sequence $\left\{\left|\varphi_{n}\right|\right\}$ is bounded on every open ball $B\left(\xi_{0}, r\right) \subset U$. Hence, on every $\overline{B\left(\xi_{0}, r^{\prime}\right)}$, with $r^{\prime}<r$, the sequence $\left\{\varphi_{n}\right\}$ is uniformly Lipschitz. Consequently, the set $\left\{\varphi_{n}\right\}_{n \in \mathbf{N}}$ is locally uniformly Lipschitz-continuous in $U$ (hence locally relatively compact in $C(U, \mathbf{R})$ ).

There is a converse in finite-dimensional spaces:

Lemma 2.18. Let $X$ be finite-dimensional, $\varphi_{n}$ and $\varphi$ be continuous convex functions on $X$ such that $\varphi_{n}(\xi) \rightarrow \varphi(\xi)$ for every $\xi \in X$. If the $\varphi_{n}$ 's are uniformly locally bounded above in $X$, then $\varphi_{n} \stackrel{\mathcal{M}}{\longrightarrow} \varphi$.

Following Brezis [4] in the Hilbert space case (see also Attouch [2]), the convergence of maximal monotone graphs is defined as follows:

Definition 2.19. Let $A_{n}, A \subset X \times X^{\prime}$ be maximal monotone graphs. The sequence $\left(A_{n}\right)_{n \geq 1}$ converges to $A$ as maximal monotone graphs when $n \rightarrow \infty$ (denoted $A_{n} \longmapsto A$ ), if for every $(\xi, \eta)$ element of $A$ there exists a sequence $\left(\xi_{n}, \eta_{n}\right)$ in $A_{n}$ such that $\left(\xi_{n}, \eta_{n}\right) \rightarrow(\xi, \eta)$ strongly in $X \times X^{\prime}$ as $n \rightarrow \infty$. 
The convergence of graphs ensures that weak limits of elements of $A_{n}$ are in $A$ provided the duality product of the pairs is controlled at the limit. More precisely,

Theorem 2.20. Let $A_{n}, A \subset X \times X^{\prime}$ be maximal monotone graphs, and let $\left(\xi_{n}, \eta_{n}\right) \in A_{n}$ and $(\xi, \eta) \in X \times X^{\prime}$. If, as $n \rightarrow+\infty$, then

$$
A_{n} \longmapsto A, \quad \xi_{n} \rightarrow \xi \quad \text { weakly in } X, \quad \eta_{n} \rightarrow \eta \quad \text { weakly in } X^{\prime},
$$

(i) if $(\xi, \eta) \in A$, then $\limsup _{n \rightarrow+\infty}\left\langle\eta_{n}, \xi_{n}\right\rangle \geq\langle\eta, \xi\rangle$;

(ii) if $\liminf _{n \rightarrow+\infty}\left\langle\eta_{n}, \xi_{n}\right\rangle \leq\langle\eta, \xi\rangle$, then $(\xi, \eta) \in A$ and $\liminf _{n \rightarrow+\infty}\left\langle\eta_{n}, \xi_{n}\right\rangle=\langle\eta, \xi\rangle$.

It noteworthy that the set of subdifferentials of lower-semicontinuous proper convex functions is closed for the convergence of maximal monotone operators:

Proposition 2.21. Let $\varphi_{n}$ be lower semicontinuous proper convex functions on $X$. If $\partial \varphi_{n} \longmapsto A$, then $A=\partial \varphi$ for some proper lower semicontinuous convex function $\varphi$ on $X$.

Proof. The proof follows from the fact that maximal and cyclic monotonicity characterizes subdifferentials and is stable under graph-convergence.

Let us now state the main result of this section, which concerns the equivalence between Mosco convergence of convex functions and the convergence of their subdifferentials, and generalizes to Banach spaces the result of [2] in the Hilbert space setting.

Theorem 2.22. If $\varphi_{n}, \varphi$ are proper lower semicontinuous convex functions on $X$, the following assertions are equivalent:

(a) $\varphi_{n} \stackrel{\mathcal{M}}{\longrightarrow} \varphi$.

(b) $\varphi_{n}^{*} \stackrel{\mathcal{M}}{\longrightarrow} \varphi^{*}$.

(c) For every $\lambda>0,\left(\varphi_{n}\right)_{\lambda} \stackrel{\mathcal{M}}{\longrightarrow} \varphi_{\lambda}$.

(d) For some $\lambda_{0}>0,\left(\varphi_{n}\right)_{\lambda_{0}} \stackrel{\mathcal{M}}{\longrightarrow} \varphi_{\lambda_{0}}$.

(e) For every $\lambda>0$, there exist $a_{\lambda}, b_{\lambda} \in \mathbf{R}^{+}$such that

$$
\forall n \in \mathbf{N}, \forall \xi \in X,\left(\varphi_{n}\right)_{\lambda}(\xi)+a_{\lambda}\|\xi\|+b_{\lambda} \geq 0
$$

and for every strongly converging sequence $\left\{\xi_{n}\right\}_{n \in \mathbf{N}}$ in $X$, with limit $\xi$

$$
\begin{aligned}
\left(\varphi_{n}\right)_{\lambda}\left(\xi_{n}\right) & \rightarrow \varphi_{\lambda}(\xi) \\
J_{\lambda}^{\partial \varphi_{n}}\left(\xi_{n}\right) & \rightarrow J_{\lambda}^{\partial \varphi}(\xi) \text { in } X \\
& \text { (equivalently } \left.\partial \varphi_{n_{\lambda}}\left(\xi_{n}\right) \rightarrow \partial \varphi_{\lambda}(\xi)\right) .
\end{aligned}
$$


(f) There exist $\lambda_{0}>0, a_{\lambda_{0}}, b_{\lambda_{0}} \in \mathbf{R}^{+}$such that

$$
\forall n \in \mathbf{N}, \forall \xi \in X,\left(\varphi_{n}\right)_{\lambda_{0}}(x)+a_{\lambda_{0}}\|\xi\|+b_{\lambda_{0}} \geq 0,
$$

$\forall \xi \in X, J_{\mu}^{\partial \varphi_{n}}(\xi) \rightarrow J_{\mu}^{\partial \varphi}(\xi)$ in $X$ (equivalently $\left.\partial \varphi_{n_{\lambda}}(\xi) \rightarrow \partial \varphi_{\lambda}(\xi)\right)$

and there exists one strongly converging sequence $\zeta_{n} \rightarrow \zeta$ in $X$, such that

$$
\left(\varphi_{n}\right)_{\lambda_{0}}\left(\zeta_{n}\right) \rightarrow_{n \rightarrow \infty} \varphi_{\lambda_{0}}(\zeta)
$$

(g) for every $\xi \in X$ and $\eta \in X^{\prime}$, there exists sequences $\left(\xi_{n}\right)_{n \geq 1}$ in $X$ and $\left(\eta_{n}\right)_{n \geq 1}$ in $X^{\prime}$ such that

$$
\xi_{n} \rightarrow \xi, \quad \limsup _{n \rightarrow \infty} \varphi_{n}\left(\xi_{n}\right) \leq \varphi(\xi), \quad \eta_{n} \rightarrow \eta, \quad \limsup _{n \rightarrow \infty} \varphi_{n}^{*}\left(\eta_{n}\right) \leq \varphi^{*}(\eta) .
$$

(h) $\forall(\xi, \eta) \in \partial \varphi$, there exists $\left(\xi_{n}, \eta_{n}\right) \in \partial \varphi_{n}$ such that $\left(\xi_{n}, \eta_{n}\right) \rightarrow(\xi, \eta)$ strongly in $X \times X^{\prime}$ and $\varphi_{n}\left(\xi_{n}\right) \rightarrow \varphi(\xi)$ and $\varphi_{n}^{*}\left(\eta_{n}\right) \rightarrow \varphi^{*}(\eta)$.

(i) $\partial \varphi_{n} \longmapsto \partial \varphi$ and there exists $(\xi, \eta) \in \partial \varphi,\left(\xi_{n}, \eta_{n}\right) \in \partial \varphi_{n}$ such that $\left(\xi_{n}, \eta_{n}\right) \rightarrow(\xi, \eta)$, strongly in $X \times X^{\prime}$ and $\varphi_{n}\left(\xi_{n}\right) \rightarrow \varphi(\xi)$.

(j) $\partial \varphi_{n} \longmapsto \partial \varphi$ and there exists $\alpha \in X$ and $\beta \in X^{\prime}$ and sequences $\left(\alpha_{n}\right)$ in $X$ and $\left(\beta_{n}\right) \in X^{\prime}$ such that

$$
\begin{array}{ll}
\alpha_{n} \rightarrow \alpha, & \limsup _{n \rightarrow \infty} \varphi_{n}\left(\alpha_{n}\right) \leq \varphi(\alpha)<\infty, \\
\beta_{n} \rightarrow \beta, & \limsup _{n \rightarrow \infty} \varphi_{n}^{*}\left(\beta_{n}\right) \leq \varphi^{*}(\beta)<\infty .
\end{array}
$$

Proof. The proof goes in a succession of implications.

- (a) $\Longrightarrow$ (D).

Fix any $\lambda>0$ and let $\xi_{n} \rightarrow \xi$ in $X$. Set $\zeta=J_{\lambda}^{\partial \varphi}(\xi)$. By the definition of Mosco convergence for $\varphi_{n}$, there exists a sequence $\left(\zeta_{n}\right)_{n \geq 1}$ in $X$ such that $\zeta_{n} \rightarrow \zeta$ and $\lim _{n \rightarrow \infty} \varphi_{n}\left(\zeta_{n}\right)=\varphi(\zeta)$. Since $\left(\varphi_{n}\right)_{\lambda}\left(\xi_{n}\right) \leq$ $\varphi_{n}\left(\zeta_{n}\right)+\frac{\left\|\xi_{n}-\zeta_{n}\right\|^{2}}{2 \lambda}$, it follows

$$
\limsup _{n \rightarrow \infty}\left(\varphi_{n}\right)_{\lambda}(\xi) \leq \varphi(\zeta)+\frac{\|\xi-\zeta\|^{2}}{2 \lambda}=\varphi_{\lambda}(\xi),
$$

which proves (M-i]) in the definition of Mosco convergence for $\left(\varphi_{n}\right)_{\lambda}$. Assume now that $\xi_{n} \rightarrow \xi$. Let $\left(\xi_{n}\right)_{\lambda}=J_{\lambda}^{\partial \varphi_{n}}\left(\xi_{n}\right)$, so

$$
\left(\varphi_{n}\right)_{\lambda}\left(x_{n}\right)=\varphi_{n}\left(\left(\xi_{n}\right)_{\lambda}\right)+\frac{\left\|x_{n}-\left(\xi_{n}\right)_{\lambda}\right\|^{2}}{2 \lambda} .
$$


Let $\zeta$ be any vector such that $\varphi(\zeta)<\infty$ and $\zeta_{n}$ be given by property $(\mathrm{M}-\mathrm{i})$ of the Mosco convergence of $\varphi_{n}$ to $\varphi$, i.e. $\zeta_{n} \rightarrow \zeta$ and $\varphi_{n}\left(\zeta_{n}\right) \rightarrow$ $\varphi(\zeta)$. In view of the inequality

$$
\varphi_{n}\left(\left(\xi_{n}\right)_{\lambda}\right)+\frac{\left\|\xi_{n}-\left(\xi_{n}\right)_{\lambda}\right\|^{2}}{2 \lambda} \leq \varphi_{n}\left(\zeta_{n}\right)+\frac{\left\|x_{n}-\zeta_{n}\right\|^{2}}{2 \lambda},
$$

and the inequality given by Proposition 2.16, we deduce that

$$
\frac{\left\|\xi_{n}-\left(\xi_{n}\right)_{\lambda}\right\|^{2}}{2 \lambda} \leq \varphi_{n}\left(\zeta_{n}\right)+\frac{\left\|\xi_{n}-\zeta_{n}\right\|^{2}}{2 \lambda}+a\left\|\left(\xi_{n}\right)_{\lambda}\right\|+b
$$

so that the sequence $\left(\left(\xi_{n}\right)_{\lambda}\right)_{n \geq 1}$ is bounded.

Consider a subsequence $\left(\left(\xi_{n_{k}}\right)_{\lambda}\right)_{k \geq 1}$ such that $\left(\xi_{n_{k}}\right)_{\lambda} \rightarrow \zeta$ and $\left(\varphi_{n_{k}}\right)_{\lambda}\left(\left(\xi_{n_{k}}\right)_{\lambda}\right) \rightarrow \liminf _{n \rightarrow \infty}\left(\varphi_{n}\right)_{\lambda}\left(\left(\xi_{n}\right)_{\lambda}\right)$. Going to the limit in (2.9), using property (M-ii) of the Mosco convergence of $\varphi_{n}$ to $\varphi$ and the weak lower semicontinuity of the norm on $X$ yields

$$
\begin{array}{r}
\liminf _{n \rightarrow \infty}\left(\varphi_{n}\right)_{\lambda}\left(\xi_{n}\right) \geq \liminf _{n \rightarrow \infty} \varphi\left(\left(\xi_{n}\right)_{\lambda}\right)+\liminf _{n \rightarrow \infty} \frac{\left\|\xi_{n}-\left(\xi_{n}\right)_{\lambda}\right\|^{2}}{2 \lambda} \\
\geq \varphi(\zeta)+\frac{\|\xi-\zeta\|^{2}}{2 \lambda} \geq \varphi_{\lambda}(\xi),
\end{array}
$$

which is (M-iii) for the sequence $\left\{\left(\varphi_{n}\right)_{\lambda}\right\}$.

- (d) $\Longrightarrow$ (d) is obvious.

- (a) $\Longrightarrow$ (的).

The inequality follows from Remark 3 .

The convergence of $\left(\varphi_{n}\right)_{\lambda}\left(\xi_{n}\right)$ to $\varphi_{\lambda}(\xi)$ when $\xi_{n} \rightarrow \xi$ follows from (d) and Lemma 2.17, since, as we show now, a local uniform upper bound exists for the family $\left(\varphi_{n}\right)_{\lambda}$. By hypothesis, there exists a sequence $\left(\xi_{n}\right)$ in $X$ which converges strongly to some $\xi$ and such that $\left(\varphi_{n}\right)\left(\xi_{n}\right) \rightarrow$ $\varphi(\xi)$ in $\mathbf{R}$. Then, by the definition of $\left(\varphi_{n}\right)_{\lambda}$ it follows that $\left(\varphi_{n}\right)_{\lambda}(\zeta) \leq$ $\varphi_{n}\left(\xi_{n}\right)+\frac{1}{\lambda}\left\|\zeta-\xi_{n}\right\|^{2}$ for every $y$ in $X$. This inequality yields the desired local uniform upper bound.

We now prove the convergence of $J_{\lambda}^{\partial \varphi_{n}}\left(\xi_{n}\right)$ to $J_{\lambda}^{\partial \varphi}(\xi)$. Set $\zeta_{n}=$ $J_{\lambda}^{\partial \varphi_{n}}\left(\xi_{n}\right)$. Then, $\left(\varphi_{n}\right)_{\lambda}\left(\xi_{n}\right)=\varphi_{n}\left(\zeta_{n}\right)+\frac{1}{2 \lambda}\left\|\xi_{n}-\zeta_{n}\right\|^{2}$. As before one can see that $\zeta_{n}$ is bounded in $X$. One can extract a weakly convergent subsequence : $\zeta_{n_{k}} \rightarrow \zeta$. From (M-ii) for the sequence $\varphi_{n}$, $\liminf \operatorname{in}_{k \rightarrow \infty} \varphi_{n_{k}}\left(\zeta_{n_{k}}\right) \geq \varphi(\zeta)$, while by weak lower semi-continuity of the norm, $\liminf \inf _{k \rightarrow \infty}\left\|\xi_{n}-\zeta_{n_{k}}\right\| \geq\|\xi-\zeta\|$. However, by the previous convergence, $\left(\varphi_{n}\right)_{\lambda}\left(\xi_{n}\right)=\varphi_{n}\left(\zeta_{n}\right)+\frac{1}{2 \lambda}\left\|\xi_{n}-\zeta_{n}\right\|^{2}$ converges to $\varphi_{\lambda}(\xi)$ which is bounded above by $\varphi(\zeta)+\frac{1}{2 \lambda}\|\xi-\zeta\|^{2}$. As a consequence, $\lim _{k \rightarrow \infty} \varphi_{n_{k}}\left(\zeta_{n_{k}}\right)=\varphi(\zeta), \lim _{k \rightarrow \infty}\left\|\xi_{n}-\zeta_{n_{k}}\right\|=\|\xi-\zeta\|$ and 
$\varphi_{\lambda}(\xi)=\varphi(\zeta)+\frac{1}{2 \lambda}\|\xi-\zeta\|^{2}$. Therefore, $\zeta=J_{\lambda}^{\partial \varphi}(\xi)$, and the whole sequence $\zeta_{n}$ converges strongly to $\zeta$ in $X$, by the local uniform convexity of the norm.

- (目) $\Longrightarrow$ (由) is obvious.

- (d) (for some $\left.\lambda_{0}>0\right) \Longrightarrow$ (困) for all $\lambda>\lambda_{0}$.

Just apply the previous (a) $\Longrightarrow$ (目) to the sequence $\left(\varphi_{n}\right)_{\lambda_{0}}$ together with Proposition 2.9.

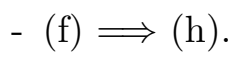

First note that by integration of the Fréchet derivative $\partial \varphi_{\lambda_{0}}$ starting at the point $x_{0}$, (迪) implies the simple convergence $\left(\varphi_{n}\right)_{\lambda_{0}}$ to $\varphi_{\lambda_{0}}$.

Let $(\xi, \eta) \in \partial \varphi$ and $\zeta=\xi+\lambda_{0} F^{-1}(\eta)$. By construction, $\xi=J_{\lambda_{0}}^{\partial \varphi}(\zeta)$. Put $\xi_{n}=J_{\lambda_{0}}^{\partial \varphi_{n}}(\zeta)$ and $\eta_{n}=\lambda_{0}^{-1} F\left(\zeta-\xi_{n}\right)=\partial\left(\varphi_{n}\right)_{\lambda_{0}}$. It is clear that $\left(\xi_{n}, \eta_{n}\right) \in \partial \varphi_{n}$. By hypothesis (田), $\xi_{n}$ converges strongly to $\xi$ in $X$, and by the bi-continuity of $F$, so does $\eta_{n}$ to $\eta$ in $X^{\prime}$.

Now, since $\varphi_{n}\left(\xi_{n}\right)=\left(\varphi_{n}\right)_{\lambda_{0}}(\zeta)-\frac{\left\|\zeta-\xi_{n}\right\|^{2}}{2 \lambda_{0}}$, for $n \rightarrow \infty$ it converges to $\varphi_{\lambda_{0}}(\zeta)-\frac{\|\zeta-\xi\|^{2}}{2 \lambda_{0}}$ which is just $\varphi(\xi)$. Finally, by Proposition 2.11, $\varphi_{n}^{*}\left(\eta_{n}\right)=\left\langle\xi_{n}, \eta_{n}\right\rangle-\varphi_{n}\left(\xi_{n}\right)$, converging to $\langle\xi, \eta\rangle-\varphi(\xi)$ which is just $\varphi^{*}(\eta)$.

- (四) $\Longrightarrow$ (耼) is obvious .

- (伍) $\Longrightarrow$ (i).

Indeed if (1i) holds, take $\alpha_{n}=\xi_{n}, \beta_{n}=\eta_{n}$. Then, by Proposition 2.11, $\varphi_{n}^{*}\left(\beta_{n}\right)=\left\langle\beta_{n}, \alpha_{n}\right\rangle-\varphi_{n}\left(\alpha_{n}\right)$ converges to $\langle\beta, \alpha\rangle-\varphi(\alpha)=\varphi^{*}(\beta)$.

- (ii) $\Longrightarrow$ (回).

Start first with $(\xi, \eta)$ be in $\partial \varphi$. For $\ell \geq 1$, set $\left(\xi^{1}, \eta^{1}\right)=(\xi, \eta)$ and let $\left(\xi^{i}, \eta^{i}\right) \in \partial \varphi$ for $2 \leq i \leq \ell$. By assumption, there exists $\left(\xi_{n}^{i}, \eta_{n}^{i}\right) \in \partial \varphi_{n}$ such that $\left(\xi_{n}^{i}, \eta_{n}^{i}\right) \rightarrow(\xi, \eta)$. By (2.6), one has

$$
\varphi_{n}\left(\xi_{n}\right) \leq \varphi_{n}\left(\alpha_{n}\right)-\sum_{i=1}^{\ell}\left\langle\eta_{n}^{i}, \xi_{n}^{i+1}-\xi_{n}^{i}\right\rangle,
$$

where $\xi_{n}^{\ell+1}=\alpha$. As $n \rightarrow \infty$, one obtains

$$
\limsup _{n \rightarrow \infty} \varphi_{n}\left(\xi_{n}\right) \leq \varphi(\alpha)-\sum_{i=1}^{\ell}\left\langle\eta_{n}, \xi^{i+1}-\xi^{i}\right\rangle .
$$

Since $\ell \geq 1$ and $\left(\xi^{i}, \eta^{i}\right)_{2 \leq i \leq \ell}$ are arbitrary, this reads

$$
\limsup _{n \rightarrow \infty} \varphi_{n}\left(\xi_{n}\right) \leq \varphi(\xi) .
$$


If $\xi \in D(\varphi) \backslash D(\partial \varphi)$, we can now use Lemma 2.4 to approximate $\xi$ by a sequence $\xi_{n}^{\prime}$ such that $\varphi\left(\xi_{n}^{\prime}\right) \rightarrow \varphi(\xi)$, then use the preceeding result for each $\xi_{n}^{\prime}$ in order to construct the sequence $\left(\xi_{n}\right)_{n \in \mathbf{N}}$ which satisfies $\lim \sup _{n \rightarrow \infty} \varphi_{n}\left(\xi_{n}\right) \leq \varphi(\xi)$.

Finally, for $\xi \in X \backslash D(\varphi), \varphi(\xi)=+\infty$ and there is nothing to prove. Since the hypothesis is symmetric under convex conjugation, (g) follows.

- (g) $\Longrightarrow($ (日) .

It suffices to prove (M-iii) in the definition of Mosco convergence. Assume that $\xi_{n} \rightarrow \xi$. For $\eta \in X^{\prime}$, let $\left(\eta_{n}\right)_{n \geq 1}$ in $X^{\prime}$ be given by the assumption (g). One has

$$
\langle\eta, \xi\rangle-\varphi^{*}(\eta) \leq \liminf _{n \rightarrow \infty}\left(\left\langle\eta_{n}, \xi_{n}\right\rangle-\varphi_{n}^{*}\left(\eta_{n}\right)\right) \leq \liminf _{n \rightarrow \infty} \varphi_{n}\left(\xi_{n}\right) .
$$

Taking the supremum over $\eta \in X^{\prime}$ allows to conclude by Definition 2.10 .

- (B) $\Longleftrightarrow(\mathrm{B})$

We know that (h) and (因) are equivalent. Since (An) is invariant under convex conjugation, it is also equivalent to (B).

Remark 5. Theorem 2.22 holds as well when $\left(\varphi_{n}\right)_{\lambda}$ and $\varphi_{\lambda}$ are replaced by $\left(\varphi_{n}\right)_{\lambda, p}$ and $\varphi_{\lambda, p}$ respectively for some fixed $p$ in $(1, \infty)$.

Remark 6. It would be tempting to consider the statement equivalent to

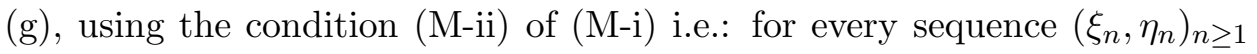
in $X \times X^{\prime}$ such that $\xi_{n} \rightarrow \xi \in X$ weakly in $X, \eta_{n} \rightarrow \eta \in X^{\prime}$ weakly in $X^{\prime}$

$$
\liminf _{n \rightarrow+\infty} \varphi_{n}\left(\xi_{n}\right) \geq \varphi(\xi), \quad \liminf _{n \rightarrow+\infty} \varphi_{n}^{*}\left(\eta_{n}\right) \geq \varphi^{*}(\eta) .
$$

However, this is not equivalent to the Mosco convergence, as can be seen from the following (counter)example: put

$$
\varphi_{n}(\xi)=n\langle\beta, \xi\rangle+n^{2},
$$

with $\beta \in X^{\prime}, \beta \neq 0$. One can check that if $\xi_{n} \rightarrow \xi$ weakly in $X$ and $\eta_{n} \rightarrow \eta$ weakly in $X^{\prime}$, then

$$
\varphi_{n}\left(\xi_{n}\right) \rightarrow+\infty \text { and } \varphi_{n}^{*}\left(\eta_{n}\right) \rightarrow+\infty \text { as } n \rightarrow+\infty .
$$

Therefore, for every proper lower semicontinuous convex function $\varphi$, for every sequence $\left(\xi_{n}, \eta_{n}\right)_{n>1}$ in $X \times X^{\prime}$ such that $\xi_{n} \rightarrow \xi \in X$ weakly in $X$, $\eta_{n} \rightarrow \eta \in X^{\prime}$ weakly in $X^{\prime}$

$$
\liminf _{n \rightarrow+\infty} \varphi_{n}\left(\xi_{n}\right) \geq \varphi(\xi), \quad \liminf _{n \rightarrow+\infty} \varphi_{n}^{*}\left(\eta_{n}\right) \geq \varphi^{*}(\eta) .
$$

But $\varphi_{n} \stackrel{\mathcal{M}}{\longrightarrow} \varphi$ is definitely not true . 


\subsection{Link between Mosco and $\Gamma$-convergences}

We first recall the definition of $\Gamma$-convergence for convex functionals, which was introduced in [15] for general functions.

Definition 2.23. Let $\varphi_{n}$ and $\varphi$ be proper lower semicontinuous convex functions. We say that $\left(\varphi_{n}\right)_{n} \Gamma$-converges to $\varphi$ for the strong topology of $X$, and we note $\varphi_{n} \stackrel{s-\Gamma}{\longrightarrow} \varphi$ if

(i) For every $\xi \in X$, there exists a sequence $\left(\xi_{n}\right)_{n \geq 1}$ converging strongly in $X$ to $\xi$ such that

$$
\limsup _{n \rightarrow+\infty} \varphi_{n}\left(\xi_{n}\right) \leq \varphi(\xi)
$$

(ii) For any sequence $\left(\xi_{n}\right)_{n \geq 1}$ of $X$ converging strongly to some $\xi$ in $X$, the following lower-bound inequality holds:

$$
\liminf _{n \rightarrow+\infty} \varphi_{n}\left(\xi_{n}\right) \geq \varphi(\xi) .
$$

The $\Gamma$-convergence for the weak topology is defined similarly.

Definition 2.24. Let $\varphi_{n}$ and $\varphi$ be proper lower semicontinuous convex functions. We say that $\left(\varphi_{n}\right)_{n} \Gamma$-converges for the weak topology of $X$ to $\varphi$, and we note $\varphi_{n} \stackrel{w-\Gamma}{\longrightarrow} \varphi$ if

(i) For every $\xi \in X$, there exists a sequence $\left(\xi_{n}\right)_{n \geq 1}$ converging weakly in $X$ to $\xi$ such that

$$
\limsup _{n \rightarrow+\infty} \varphi_{n}\left(\xi_{n}\right) \leq \varphi(\xi) .
$$

(ii) For any sequence $\left(\xi_{n}\right)_{n \geq 1}$ of $X$ converging weakly to some $\xi$ in $X$, the following lower-bound inequality holds:

$$
\liminf _{n \rightarrow+\infty} \varphi_{n}\left(\xi_{n}\right) \geq \varphi(\xi) .
$$

Remark 7 . The notion of $\Gamma$-convergence is well-adapted to study the limit of variational problems. It is weaker than the Mosco convergence. We refer to [1] and [11] for more details.

Remark 8. Let $\varphi_{n}$ and $\varphi$ be proper lower semicontinuous convex functions. The following equivalence is straightforward:
(a) $\varphi_{n} \stackrel{\mathcal{M}}{\longrightarrow} \varphi \Longleftrightarrow$
(b) $\varphi_{n} \stackrel{s-\Gamma}{\longrightarrow} \varphi$ and $\varphi_{n} \stackrel{w-\Gamma}{\longrightarrow} \varphi$.

Actually, more can be said when considering also the conjugates.

Proposition 2.25. Let $\varphi_{n}$ and $\varphi$ be proper lower semicontinuous convex functions. The following statements are equivalent. 
(1) $\varphi_{n} \stackrel{\mathcal{M}}{\longrightarrow} \varphi$.

(2) $\varphi_{n} \stackrel{s-\Gamma}{\longrightarrow} \varphi$ and $\varphi_{n}^{*} \stackrel{s-\Gamma}{\longrightarrow} \varphi^{*}$.

Moreover, either statement implies both $\varphi_{n} \stackrel{w-\Gamma}{\longrightarrow} \varphi$ and $\varphi_{n}^{*} \stackrel{w-\Gamma}{\longrightarrow} \varphi^{*}$.

Proof. Recalling Theorem 2.22 (a) and (B) together with Remark 8 we obtain that (1) implies to (2). The converse follows from the equivalence between (G) and (日) in Theorem 2.22 .

Remark 9. When $X$ is infinite dimensional, if $\varphi_{n} \stackrel{s-\Gamma}{\longrightarrow} \varphi$ and $\varphi_{n}^{*} \stackrel{s-\Gamma}{\longrightarrow} \psi$, this does not necessarly imply the Mosco convergence, since $\psi \neq \varphi^{*}$ can occur.

Indeed, consider

$$
\varphi_{n}(\xi)=\frac{\left\|\xi-\alpha_{n}\right\|^{2}}{2}
$$

where $\left\|\alpha_{n}\right\|=1$ and $\alpha_{n} \rightarrow 0$. One has

$$
\varphi_{n} \stackrel{s-\Gamma}{\longrightarrow} \varphi \text { and } \varphi_{n}^{*} \stackrel{s-\Gamma}{\longrightarrow} \psi
$$

with $\varphi(\xi)=\frac{\|\xi\|^{2}+1}{2}$ and $\psi(\eta)=\frac{\|\eta\|^{2}}{2}$.

The same example also shows that, one can have $\varphi_{n} \stackrel{w-\Gamma}{\longrightarrow} \varphi_{1}$ and $\varphi_{n}^{*} \stackrel{w-\Gamma}{\longrightarrow} \psi_{1}, \psi_{1} \neq \varphi_{1}^{*}$, with $\varphi_{1}(\xi)=\frac{\|\xi\|^{2}}{2}$ and $\psi_{1}(\eta)=\frac{\|\eta\|^{2}-1}{2}$. Indeed, let us consider a sequence $\left(\xi_{n}\right)$ which weakly converges to $\xi$ in $X$, then $\xi_{n}-\alpha_{n} \rightarrow \xi$ and by weak lower semicontinuity, $\lim \left\|\xi_{n}-\alpha_{n}\right\| \geq \frac{\|\xi\|^{2}}{2}$. For the recovery sequence, let us take $\xi_{n}=\xi+\alpha_{n}$, then $\varphi_{n}\left(\xi_{n}\right)=\frac{\|\xi\|^{2}}{2}$. The same can be done for the conjugate function.

It is not clear whether $\varphi_{n} \stackrel{w-\Gamma}{\longrightarrow} \varphi$ and $\varphi_{n}^{*} \stackrel{w-\Gamma}{\longrightarrow} \varphi^{*}$ implies the convergence of $\varphi_{n}$ to $\varphi$ in the sense of Mosco.

\section{The canonical extension of a maximal monotone graph and the integration of a normal convex integrand}

\subsection{Measurability}

The study of functionals of the form

$$
I_{\varphi}(u)=\int_{\Omega} \varphi(t, u(t)) \mathrm{d} \mu,
$$

requires the understanding of the measurability of families of convex maps depending on a parameter in a measure space and of the corresponding families of maximal monotone operators. We will prove that when $A(t)$ 
is the subdifferential of a continuous proper lower semicontinuous convex function $\varphi(t, \cdot)$, the measurability of $A$ is equivalent to the measurability of $\varphi(\cdot, \xi)$.

In this section, $(\Omega, \mathcal{T}, \mu)$ is a finite or $\sigma$-finite measure space and the space $X$ is assumed to be separable. Since $X$ is reflexive, $X^{\prime}$ will also be separable. The set of maximal monotone operators from $X$ to $X^{\prime}$ is denoted by $\mathfrak{M}\left(X \times X^{\prime}\right)$.

We shall consider maps whose values are maximal monotone operators or convex functions as special cases of multivalued operators. The measurability of such maps is defined according to [7].

Definition 3.1. Let $(M, d)$ be a separable metric space and $(\Omega, \mathcal{T})$ is a measurable space. The map $\Gamma: \Omega \rightarrow \wp(M)$ is measurable if for every open set $U \subset M$, the set

$$
\{t \in \Omega: \Gamma(t) \cap U \neq \emptyset\}
$$

is measurable.

Remark 10. One readily sees that if

$$
\{t \in \Omega: \Gamma(t) \cap B \neq \emptyset\}
$$

is measurable for every Borel set $B$ (resp. for every closed set, open balls or closed balls), then $\Gamma$ is measurable since any open set can be written as a countable union of such sets. The completeness of the measure-space $(\Omega, \mathcal{T}, \mu)$ implies that these stronger definitions are in fact equivalent (see [7, chapter III]).

There are several equivalent characterization of measurable multivalued mappings.

Theorem 3.2 (Castaing and Valadier [7]). Let $(M, d)$ be a separable metric space, $(\Omega, \mathcal{T})$ be a measurable space and $\Gamma: \Omega \rightarrow \wp(M)$. If for every $t \in \Omega, \Gamma(t)$ is closed and not empty, then the following properties are equivalent

(a) $\Gamma$ is measurable,

(b) for every $x \in M, t \mapsto d(x, \Gamma(t)) \doteq \inf _{y \in \Gamma(t)} d(x, y)$ is measurable,

(c) there exists a countable family of measurable mappings $\sigma_{n}: \Omega \rightarrow M$, $n \in \mathbf{N}$, called measurable sections, such that for every $t \in \Omega$,

$$
\Gamma(t)=\overline{\left\{\sigma_{n}(t): n \in \mathbf{N}\right\}} .
$$


Going back to the measurability of maximal monotone operators, the map $A: \Omega \rightarrow \mathfrak{M}\left(X \times X^{\prime}\right)$ is measurable if it is measurable as a multivalued map from $\Omega$ to $\wp\left(X \times X^{\prime}\right)$.

Similarly, the measurability of convex function-valued maps can be defined in terms of their epigraph, hence the following definition:

Definition 3.3 (Rockafellar [23], [24]). Let $\varphi: \Omega \times X \rightarrow \mathbf{R} \cup\{+\infty\}$ be such that $\varphi(t, \cdot)$ is a proper lower semicontinuous function. The function $\varphi$ is a normal convex integrand if the map $t \in \Omega \mapsto \operatorname{epi} \varphi(t, \cdot) \subset \wp(X \times \mathbf{R} \cup\{+\infty\})$ is measurable.

Example 3. If $\varphi: \Omega \times X \rightarrow \mathbf{R}$ is Carathéodory and convex, i.e. $\varphi(t, \cdot)$ is a continuous convex function for every $t \in \Omega$ and $\varphi(\cdot, \xi)$ is measurable for every $\xi$, then it is a normal convex integrand. Indeed, if $\left(\xi_{n}\right)_{n \geq 1}$ is dense in $X$ and $\left(q_{m}\right)$ is dense in $\mathbf{R}^{+}$, one can reconstruct the epigraph map from the countable family of measurable sections

$$
\left\{\varphi\left(t, \xi_{n}\right)+q_{m}\right\}, n, m \in \mathbf{N} .
$$

On the other hand, Carathéodory functions can be used to construct normal integrands, as the following proposition shows.

Proposition 3.4. The supremum of a countable family of Carathéodory convex functions which is proper is a normal integrand.

Proof. It follows directly from Definition 3.1 of measurability.

We are now in position to state the main result of this section.

Theorem 3.5. Let $X$ be a reflexive separable Banach space and let $\varphi$ : $\Omega \times X \rightarrow \mathbf{R} \cup\{+\infty\}$. If $\varphi(t, \cdot)$ is a lower semicontinuous proper convex function for almost every $t$ in $\Omega$, then the following are equivalent:

(a) $\varphi$ is a normal convex integrand,

(b) $\varphi^{*}$ is a normal convex integrand,

(c) for every $\lambda>0, \varphi_{\lambda}$ is a convex Carathéodory function,

(d) there exists some $\lambda_{0}>0$ such that $\varphi_{\lambda_{0}}$ is a convex Carathéodory function,

(e) for every $\lambda>0$ and every $\xi \in X$, the map $t \mapsto J_{\lambda}^{\varphi(t)}(\xi)$ is measurable from $\Omega$ to $X$ (equivalently $t \mapsto \partial \varphi(t)_{\lambda}(\xi)$ is measurable from $\Omega$ to $X^{\prime}$ ) and the map $t \mapsto \varphi(t)_{\lambda}(\xi)$ is measurable from $\Omega$ to $\mathbf{R}$,

(f) there exists some $\lambda_{0}>0$ such that for every $\xi \in X$, the map $t \mapsto J_{\lambda_{0}}^{\varphi(t)}(\xi)$ is measurable from $\Omega$ to $X$ (equivalently $t \mapsto \partial \varphi(t)_{\lambda_{0}}(\xi)$ is measurable from $\Omega$ to $X^{\prime}$ ) and there is some $\xi_{0} \in X$ such that $t \mapsto \varphi(t)_{\lambda_{0}}\left(\xi_{0}\right)$ is measurable from $\Omega$ to $\mathbf{R}$, 
(g) $\partial \varphi(t): \Omega \rightarrow \mathfrak{M}\left(X \times X^{\prime}\right)$ is measurable, and there exists measurable mapping $\alpha: \Omega \rightarrow X$ and $\beta: \Omega \rightarrow X^{\prime}$ such that $(\alpha(t), \beta(t)) \in \partial \varphi(t)$ for every $t \in \Omega$, such that the functions $t \mapsto \varphi(t, \alpha(t))$ and $t \mapsto \varphi^{*}(t, \beta(t))$ are measurable,

(h) $\partial \varphi(t): \Omega \rightarrow \mathfrak{M}\left(X \times X^{\prime}\right)$ is measurable, and there exists a measurable function $\alpha: \Omega \rightarrow X$ such that $\alpha(t) \in D(\varphi(t))$ and $t \mapsto \varphi(t, \alpha(t))$ is measurable.

Remark 11. Theorem 3.5 was proved by Attouch when $X$ is a Hilbert space [1].

Proof of Theorem 3.5.

(回) $\Longleftrightarrow$ (B):

Let $\alpha_{n}: \Omega \rightarrow X$ and $\tau_{n}: \Omega \rightarrow \mathbf{R} \cup\{+\infty\}$ be such that epi $\varphi(t)=\overline{\left\{\left(\alpha_{n}(t), \tau_{n}(t)\right): n \geq 1\right\}}$.

For $\eta \in X^{\prime}, \varphi^{*}(t, \eta)=\sup _{n \in \mathbf{N}}\left\langle y, \alpha_{n}(t)\right\rangle-\tau_{n}(t)$. It is therefore a normal convex integrand by Proposition 3.4. Therefore, (a) implies (B). Exchanging the roles of $\varphi$ and $\varphi^{*}$ proves the equivalence.

A similarly reasoning shows (a) $\Longrightarrow$ (d).

(d) $\Longrightarrow$ (d) and (目) $\Longrightarrow$ (田) are obvious.

$(\mathrm{d}) \Longrightarrow\left(\right.$ 目) and $(\mathrm{d}) \Longrightarrow(\mathbb{G})$. Since $\varphi_{\lambda}$ is Carathéodory and $C^{1}$ with respect to $\xi \in X$, it follows that for each $\xi \in X, t \mapsto \varphi_{\lambda}^{\prime}(t, \xi)$ is weakly measurable from $\Omega$ to $X^{\prime}$. Since $X$ is reflexive and separable, it follows that $X^{\prime}$ is separable, so that weak and strong measurability are equivalent.

$(\mathbb{T}) \Longrightarrow(\mathrm{g})$. Under the hypotheses of $(\mathbb{E})$, since $\xi \mapsto \varphi_{\lambda_{0}}(\xi)$ is $C^{1}$, it follows that for every $\xi \in X, t \mapsto \varphi_{\lambda_{0}}(\xi)$ is measurable.

Let now $\left\{\xi_{n}\right\}_{n \in \mathbf{N}}$ be a dense sequence in $X$. For each $n$, set $\left(\alpha_{n}(t), \beta_{n}(t)\right)$ $=\left(J_{\lambda_{0}}^{\partial \varphi(t)}\left(\xi_{n}\right), \partial \varphi_{\lambda_{0}}\left(t, \xi_{n}\right)\right)$, which are measurable.

For a.e. $t \in \Omega$, and $(\xi, \eta) \in \partial \varphi(t)$, set $\zeta=\xi+\lambda_{0} F^{-1}(\eta)$ so that the pair $(\xi, \eta)$ is $\left(J_{\lambda_{0}}^{\partial \varphi(t)}(\xi), \partial \varphi_{\lambda_{0}}(t, \xi)\right)$. For $x_{n_{k}} \rightarrow \zeta$, Lemma 2.4 implies the strong convergence of $\left(\alpha_{n_{k}}(t), \beta_{n_{k}}(t)\right)$ to $(\xi, \eta)$. This shows that $\left(\alpha_{n}(t), \beta_{n}(t)\right)$ is a countable family which is dense for $t \mapsto \partial \varphi(t)$. Since $\varphi\left(t, \alpha_{n}(t)\right)=\varphi_{\lambda_{0}}\left(t, \xi_{n}\right)-\frac{1}{2 \lambda_{0}}\left\|\xi_{n}-\alpha_{n}(t)\right\|$, it follows that $t \mapsto \varphi\left(t, \alpha_{n}(t)\right)$ is measurable. Similarly, $\varphi^{*}\left(t, \beta_{n}(t)=\left\langle\alpha_{n}(t), \beta_{n}(t)\right\rangle-\varphi\left(t, \alpha_{n}(t)\right)\right.$ is measurable.

(G) $\Longrightarrow$ (II) is obvious.

(1] $\Longrightarrow$ (19) . By Theorem 3.2 there exists measurable maps $\alpha_{n}: \Omega \rightarrow X$ and $\beta_{n}: \Omega \rightarrow X^{\prime}$ such that $\partial \varphi(t)=\overline{\left\{\left(\alpha_{n}(t), \beta_{n}(t)\right): n \geq 1\right\}}$. By (2.6),

$$
\begin{array}{r}
\varphi\left(\alpha_{n}(t)\right)(t)=\varphi(t, \alpha(t))-\sup \left\{\sum_{i=1}^{\ell}\left\langle\eta^{i}, \xi^{i+1}-\xi^{i}\right\rangle: \ell \geq 1,\left(\xi^{i}, \eta^{i}\right) \in \partial \varphi,\right. \\
\left.\xi^{1}=\alpha_{n}(t), \eta_{1}=\beta_{n}(t) \text { and } \xi^{\ell+1}=\alpha(t)\right\} .
\end{array}
$$


This function is measurable since

$$
\begin{gathered}
\sup \left\{\sum_{i=1}^{\ell}\left\langle\eta^{i}, \xi^{i+1}-\xi^{i}\right\rangle: \ell \geq 1,\left(\xi^{i}, \eta^{i}\right) \in \partial \varphi\right. \\
\left.\xi^{1}=\alpha_{n}(t), \eta_{1}=\beta_{n}(t) \text { and } \xi^{\ell+1}=\alpha(t)\right\} \\
=\sup \left\{\sum_{i=1}^{\ell}\left\langle\beta_{n_{k}}(t), \alpha_{n_{k+1}}(t)-\alpha_{n_{k+1}}\right\rangle: \ell \geq 1, n_{k} \geq 1,\right. \\
\left.n_{1}=n \text { and } \alpha_{n_{\ell+1}}=\alpha(t)\right\} .
\end{gathered}
$$

By Lemma 2.4 (and some diagonal procedure), for every $\xi \in D(\varphi)$, there exists a sequence $\left(n_{k}\right)_{k \geq 1}$ such that $\alpha_{n_{k}}(t) \rightarrow \xi$ and $\varphi\left(t, \alpha_{n_{k}}(t) \rightarrow \varphi(t, \xi)\right.$. Therefore

$$
\operatorname{epi}(\varphi(t))=\overline{\left\{\left(\alpha_{n}(t), \varphi\left(t, \alpha_{n}(t)\right)+q\right): n \geq 1, q \in \mathbf{Q}_{+}\right\}},
$$

and $\varphi$ is a normal convex integrand.

\subsection{Integrating normal convex integrands}

For a normal convex integrand $\varphi: \Omega \times X \rightarrow \mathbf{R} \cup\{+\infty\}$ and $u \in L^{p}(\Omega, X)$, how can one define

$$
I_{\varphi}(u)=\int_{\Omega} \varphi(t, u(t)) \mathrm{d} \mu ?
$$

The integral does not necessarily make sense. However, if there exists $\beta \in$ $L^{q}\left(\Omega, X^{\prime}\right)$ such that $\varphi^{*}(t, \beta(t)) \in L^{1}(\Omega, \mu)$, then by Proposition 2.11

$$
\varphi(t, u(t)) \geq\langle\beta(t), u(t)\rangle-\varphi^{*}(t, \beta(t)) .
$$

Since the latter is integrable, $\int_{\Omega} \varphi(t, u(t)) \mathrm{d} \mu$ is well-defined and convex in $\mathbf{R} \cup+\infty$. By a judicious application of Fatou's lemma, it is also lower semicontinuous.

The following theorem of Rockafellar makes the connection with conjugation in the case of $L^{p}$-spaces.

Theorem 3.6 (Rockafellar [24]). Let $\varphi$ be a normal convex integrand. If there exists $\alpha \in L^{p}(\Omega, X)$ such that $\varphi(t, \alpha(t)) \in \mathrm{L}^{1}(\Omega)$ and $\beta \in L^{q}(\Omega, X)$ such that $\varphi^{*}(t, \beta(t)) \in \mathrm{L}^{1}(\Omega)$, then $I_{\varphi}$ and $I_{\varphi^{*}}$ are proper lower semicontinuous convex functions and $\left(I_{\varphi}\right)^{*}=I_{\varphi^{*}}$.

Given a map $A: \Omega \rightarrow \mathfrak{M}\left(X \times X^{\prime}\right)$, it has a canonical extension from $L^{p}(\Omega ; X)$ to $L^{q}\left(\Omega ; X^{\prime}\right)$, which is itself monotone.

Definition 3.7. The canonical extension of $A: \Omega \rightarrow \mathfrak{M}\left(X \times X^{\prime}\right)$, from $L^{p}(\Omega ; X)$ to $L^{q}\left(\Omega ; X^{\prime}\right)$ (where $\left.1 / p+1 / q=1\right)$, is

$$
\mathcal{A}=\left\{(u, v) \in L^{p}(\Omega ; X) \times L^{q}\left(\Omega ; X^{\prime}\right):(u(t), v(t)) \in A(t) \text { a.e. } t \in \Omega\right\} .
$$


It is known (see [14]) that the operator $\mathcal{A}$ is maximal if and only if it is not empty.

In the particular case where $\varphi: \Omega \times X \rightarrow \mathbf{R} \cup\{+\infty\}$ is a normal convex integrand, the canonical extension $\mathcal{A}_{\partial \varphi}$ of $\partial \varphi$ is

$$
\mathcal{A}_{\partial \varphi}=\left\{(u, v) \in L^{p}(\Omega ; X) \times L^{q}\left(\Omega ; X^{\prime}\right):(u(t), v(t)) \in \partial \varphi(t) \text { a.e. } t \in \Omega\right\} .
$$

A natural question arises: are $\partial\left(I_{\varphi}\right)$ and $\mathcal{A}_{\partial \varphi}$ somehow connected?

Theorem 3.8. Under the assumptions of Theorem 3.6,

$$
\partial\left(I_{\varphi}\right)=\mathcal{A}_{\partial \varphi}
$$

In particular $\mathcal{A}_{\partial \varphi}$ is maximal monotone.

Proof. Let $(u, v)$ be in $\partial I_{\varphi}$. By Theorem 3.6 and Proposition 2.11,

$$
\int_{\Omega} \varphi(t, u(t))+\varphi^{*}(t, v(t)) \mathrm{d} \mu=I_{\varphi}(u)+\left(I_{\varphi}\right)^{*}(v)=\int_{\Omega}\langle v(t), u(t)\rangle \mathrm{d} \mu .
$$

But, by Young's inequality, for every $t \in \Omega$ :

$$
\varphi(t, u(t))+\varphi^{*}(t, v(t)) \geq\langle v(t), u(t)\rangle .
$$

Therefore,

$$
\varphi(t, u(t))+\varphi^{*}(t, v(t))-\langle v(t), u(t)\rangle=0, \text { for almost every } t \in \Omega,
$$

so that $(u, v) \in A_{\partial \varphi}$.

Thus, $\partial\left(I_{\varphi}\right) \subset \mathcal{A}_{\partial \varphi}$. Since $I_{\varphi}$ is lower semi-continuous, convex and proper, $\partial\left(I_{\varphi}\right)$ is maximal monotone by Proposition 2.13, and $\mathcal{A}_{\partial \varphi}$, which is monotone, must equal $\partial\left(I_{\varphi}\right)$.

Corollary 3.9. Under the assumptions of Theorem 3.9, with the natural norms on the spaces $L^{p}(\Omega ; X)$ and $L^{q}\left(\Omega ; X^{\prime}\right)$, the resolvant $J_{\lambda, p}^{\partial I_{\varphi}}$ is the canonical extension of the resolvant $t \rightarrow J_{\lambda, p}^{\partial \varphi(t)}$, and the Yosida approximation $\mathcal{A}_{\left(\partial I_{\varphi}\right)_{\lambda}}$ is the canonical extension of the Yosida approximation $t \mapsto A_{\partial \varphi(t)_{\lambda}}$. Furthermore, $\left(I_{\varphi}\right)_{\lambda, p}=I_{\varphi_{\lambda, p}}$.

Proof. If $w$ is in $L^{p}(\Omega ; X),(u, v)=\left(J_{\lambda, p}^{\partial I_{\varphi}}(w), \mathcal{A}_{\left(\partial I_{\varphi}\right)_{\lambda}}(w)\right)$ exactly means

$$
(u, v) \in \mathcal{A}_{\left(\partial I_{\varphi}\right)} \text { and } w=u+\lambda F_{L^{p}(\Omega ; X)}^{-1}(v) .
$$

But this implies that for a.e. $t \in \Omega u(t)+\lambda F_{X}^{-1}(v(t))$ and $(u(t), v(t)) \in$ $A_{(\partial I \varphi(t))}$, hence the result.

Now, 


$$
\begin{aligned}
\left(I_{\varphi}\right)_{\lambda, p}(w) & =I_{\varphi}(u)+\frac{\lambda}{p}\|w-u\|_{L^{p}(\Omega ; X)}^{p} \\
& =\int_{\Omega}\left(\varphi(t, u(t))+\frac{\lambda}{p}\|w(t)-u(t)\|_{X}^{p} \mathrm{~d} \mu\right. \\
& =\int_{\Omega} \varphi_{\lambda, p}(t, w(t)) \mathrm{d} \mu .
\end{aligned}
$$

In the previous proof, it is part of the hypothesis that $u$ is in $L^{p}(\Omega ; X)$ and $v$ in $L^{q}\left(\Omega ; X^{\prime}\right)$. However, under the hypothesis that $\varphi(t, \cdot)$ is a normal convex integrand, from Theorem 3.5 it follows that for every measurable $w(t)$ defined on $\Omega$ with values in $X$, the functions $t \rightarrow u(t) \doteq J_{\lambda, p}^{\partial \varphi(t)}(w(t))$ and $t \mapsto v(t) \doteq A_{\partial \varphi(t)}(w(t))$ are measurable. Under the extra hypothesis of Theorem 3.6, if $w$ belongs to $L^{p}(\Omega ; X)$, then so does $u$ while $v$ belongs to $L^{q}\left(\Omega ; X^{\prime}\right)$ :

Proposition 3.10. Under the assumptions of Theorem 3.0, if $w$ belongs to $L^{p}(\Omega ; X)$, then $t \rightarrow J_{\lambda, p}^{\partial \varphi(t)}(w(t))$ is in the same space, and $t \mapsto A_{\partial \varphi(t)}(w(t))$ is in $L^{q}\left(\Omega ; X^{\prime}\right)$.

Proof. From the properties of the duality mapping, the two conclusions are equivalent. We show the first one. Young's inequality implies

$$
\varphi(t, x)-\langle x, \beta(t)\rangle+\varphi^{*}(t, \beta(t)) \geq 0,
$$

so

$$
\varphi(t, x)+\|x\|_{X}\|\beta(t)\|_{X^{\prime}}+\varphi^{*}(t, \beta(t)) \geq 0 .
$$

Applying this to $u(t)=J_{\lambda, p}^{\partial I_{\varphi}}(w(t))$ together with

$$
\begin{aligned}
\varphi_{\lambda, p}(t, w(t)) & =\varphi(t, u(t))+\frac{1}{p \lambda^{p-1}}\|w(t)-u(t)\|_{X}^{p} \\
& \leq \varphi(t, \alpha(t))+\frac{1}{p \lambda^{p-1}}\|w(t)-\alpha(t)\|_{X}^{p}
\end{aligned}
$$

yields

$$
\begin{aligned}
\frac{1}{p \lambda^{p-1}}\|w(t)-u(t)\|_{X}^{p} \leq \varphi(t, \alpha(t))+\frac{1}{p \lambda^{p-1}}\|w(t)-\alpha(t)\|_{X}^{p} & \\
& +\|v(t)\|_{X}\|\beta(t)\|_{X^{\prime}}+\varphi^{*}(t, \beta(t))
\end{aligned}
$$

It follows that there exists some constant $C_{p, \lambda}$ (which only depends on $\lambda$ and $p$ ) such that for a.e. $t \in \Omega$,

$$
\begin{gathered}
\|u(t)\|_{X}^{p} \leq C_{p, \lambda}\left(\|w(t)\|_{X}^{p}+\|\alpha(t)\|_{X}^{p}+\right. \\
\left.\|\beta(t)\|_{X^{\prime}}^{q}+\varphi(t, \alpha(t))+\varphi^{*}(t, \beta(t))\right) .
\end{gathered}
$$

This implies that $u$ belongs to $L^{p}(\Omega ; X)$. 


\subsection{Convergence of canonical extensions and Mosco conver- gence of sequence of normal convex integrands}

Given functions $A, A_{n}: \Omega \rightarrow \mathfrak{M}\left(X \times X^{\prime}\right)$ and their canonical extensions $\mathcal{A}, \mathcal{A}^{n}$, the question whether the pointwise convergence $A_{n}(t) \longmapsto A(t)$ implies the convergence of the induced graphs $\mathcal{A}^{n} \longmapsto \mathcal{A}$ was considered in [14] with the following result:

Theorem 3.11. Let $A, A_{n}: \Omega \rightarrow \mathfrak{M}\left(X \times X^{\prime}\right)$ be measurable. Assume

(i) for almost every $t \in \Omega, A_{n}(t) \longmapsto A(t)$ as $n \rightarrow \infty$,

(ii) $\mathcal{A}$ and $\mathcal{A}^{n}$ are maximal monotone,

(iii) there exists $\left(\alpha_{n}, \beta_{n}\right) \in \mathcal{A}^{n}$ and $(\alpha, \beta) \in L^{p}(\Omega ; X) \times L^{q}\left(\Omega ; X^{\prime}\right)$ such that $\left(\alpha_{n}, \beta_{n}\right) \rightarrow(\alpha, \beta)$ strongly in $L^{p}(\Omega ; X) \times L^{q}\left(\Omega ; X^{\prime}\right)$ as $n \rightarrow \infty$,

then $\mathcal{A}^{n} \longmapsto \mathcal{A}$.

Recalling the results of section 2 , we see that in terms of normal convex integrands, the question becomes: given $\varphi, \varphi_{n}$ and their associated graphs $\mathcal{A}_{\partial \varphi}, \mathcal{A}_{\partial \varphi_{n}}$, does the Mosco convergence $\varphi_{n}(t,.) \stackrel{\mathcal{M}}{\longrightarrow} \varphi(t,$.$) imply the Mosco$ convergence of the induced functionals $I_{\varphi_{n}} \stackrel{\mathcal{M}}{\longrightarrow} I_{\varphi}$ and convergence of the induced graphs $\mathcal{A}_{\partial \varphi}, \mathcal{A}_{\partial \varphi_{n}}$ ?

Theorem 3.12. Let $\varphi, \varphi_{n}: \Omega \times X \rightarrow \mathbf{R} \cup\{+\infty\}$ be normal convex integrands such that for every $t \in \Omega, \varphi_{n}(t,.) \stackrel{\mathcal{M}}{\longrightarrow} \varphi(t,$.$) as n \rightarrow \infty$. Let $\alpha_{n}$ and $\alpha$ in $L^{p}(\Omega), \beta_{n}$ and $\beta$ in $L^{q}(\Omega)$ be such that $\varphi_{n}\left(t, \alpha_{n}(t)\right) \in L^{1}(\Omega)$, $\varphi_{n}^{*}\left(t, \beta_{n}(t)\right) \in L^{1}(\Omega), \varphi(t, \alpha(t)) \in L^{1}(\Omega)$ and $\varphi^{*}(t, \beta(t)) \in L^{1}(\Omega)$. If

$$
\begin{array}{ll}
\alpha_{n} \rightarrow \alpha \quad \text { in } L^{p}(\Omega, X), & \limsup _{n \rightarrow \infty} I_{\varphi_{n}}\left(\alpha_{n}\right) \leq I_{\varphi}(\alpha), \\
\beta_{n} \rightarrow \beta \quad \text { in } L^{q}\left(\Omega, X^{\prime}\right), & \limsup _{n \rightarrow \infty} I_{\varphi_{n}^{*}}\left(\beta_{n}\right) \leq I_{\varphi^{*}}(\beta),
\end{array}
$$

then

$$
I_{\varphi_{n}} \stackrel{\mathcal{M}}{\longrightarrow} I_{\varphi}
$$

In particular, $\mathcal{A}_{\partial \varphi_{n}} \longmapsto \mathcal{A}_{\partial \varphi}$.

Lemma 3.13. Under the hypotheses of Theorem 3.19, $t \mapsto \varphi_{n}\left(t, \alpha_{n}(t)\right)$ converges to $\varphi(t, \alpha(t))$ a.e. in $\Omega$ and $I_{\varphi_{n}}\left(\alpha_{n}\right)$ actually converges to $I_{\varphi}(\alpha)$. The similar statement holds true for $\varphi_{n}^{*}\left(t, \beta_{n}(t)\right)$.

Furthermore, the sequence of functions $t \mapsto\left(\varphi_{n}\left(t, \alpha_{n}(t)\right)+\varphi_{n}^{*}\left(t, \beta_{n}(t)\right)\right)$ converges to its limit a.e. $\left(\varphi(t, \alpha(t))+\varphi^{*}(t, \beta(t))\right)$ in $L^{1}(\Omega)$.

Proof. It is based on a precise application of Fatou's lemma (in its most general version which applies to a sequence bounded below by a convergent sequence in $L^{1}(\Omega)$ ). Up to a subsequence (still denoted by $\{n\}$ ), for almost 
every $t \in \Omega, \alpha_{n}(t) \rightarrow \alpha(t)$ in $X$ and $\beta_{n}(t) \rightarrow \beta(t)$ in $X^{\prime}$, so, by definition of the Mosco convergence, for a.e. $t \in \Omega$.

$$
\liminf _{n \rightarrow \infty}\left(\varphi_{n}\left(t, \alpha_{n}(t)\right)+\varphi_{n}^{*}\left(t, \beta_{n}(t)\right)\right) \geq \varphi(t, \alpha(t))+\varphi^{*}(t, \beta(t)) .
$$

Noting that $\varphi_{n}\left(t, \alpha_{n}(t)\right)+\varphi_{n}^{*}\left(t, \beta_{n}(t)\right) \geq\left\langle\alpha_{n}(t), \beta_{n}(t)\right\rangle$, where $\left\langle\alpha_{n}, \beta_{n}\right\rangle \rightarrow$ $\langle\alpha, \beta\rangle$ in $L^{1}(\Omega)$, one can apply Fatou's Lemma in order to obtain for any measurable subset $E$ in $\Omega$ :

$$
\liminf _{n \rightarrow \infty} \int_{E} \varphi_{n}\left(t, \alpha_{n}(t)\right)+\varphi_{n}^{*}\left(t, \beta_{n}(t)\right) \mathrm{d} \mu \geq \int_{E} \varphi(t, \alpha(t))+\varphi^{*}(t, \beta(t)) \mathrm{d} \mu .
$$

This is true in particular for $E=\Omega$. However, the opposite inequality is satisfied on $\Omega$ as a consequence of the last hypothesis of Theorem 3.12:

$$
\begin{gathered}
\limsup _{n \rightarrow \infty} \int_{\Omega} \varphi_{n}\left(t, \alpha_{n}(t)\right)+\varphi_{n}^{*}\left(t, \beta_{n}(t)\right) \mathrm{d} \mu \leq \limsup _{n \rightarrow \infty} I_{\varphi_{n}}\left(\alpha_{n}\right)+\limsup _{n \rightarrow \infty} I_{\varphi_{n}^{*}}\left(\beta_{n}\right) \\
\leq \int_{\Omega} \varphi(t, \alpha(t))+\varphi^{*}(t, \beta(t)) \mathrm{d} \mu=I_{\varphi}(\alpha)+I_{\varphi^{*}}(\beta) .
\end{gathered}
$$

Consequently, $\lim _{n \rightarrow \infty} I_{\varphi_{n}}\left(\alpha_{n}\right)=I_{\varphi}(\alpha)$ and $\lim _{n \rightarrow \infty} I_{\varphi_{n}^{*}}\left(\beta_{n}\right)=I_{\varphi^{*}}(\beta)$.

From these equalities together with (3.6) and Fatou's lemma again, it follows that

$$
\lim _{n \rightarrow \infty}\left(\varphi_{n}\left(t, \alpha_{n}(t)\right)+\varphi_{n}^{*}\left(t, \beta_{n}(t)\right)\right)=\varphi(t, \alpha(t))+\varphi^{*}(t, \beta(t))
$$

for a.e. $t$ in $\Omega$. Comparing with the opposite inequalities due to the Mosco convergences $\varphi_{n}(t,.) \stackrel{\mathcal{M}}{\longrightarrow} \varphi(t,$.$) and \varphi_{n}^{*}(t,.) \stackrel{\mathcal{M}}{\longrightarrow} \varphi^{*}(t,$.$) , one actually con-$ cludes that

$$
\left.\lim _{n \rightarrow \infty} \varphi_{n}\left(t, \alpha_{n}(t)\right)=\varphi(t, \alpha(t)) \quad \text { and } \quad \lim _{n \rightarrow \infty} \varphi_{n}^{*}\left(t, \beta_{n}(t)\right)\right)=\varphi^{*}(t, \beta(t))
$$

for a.e. $t$ in $\Omega$. Now, consider the nonnegative function

$$
\theta_{n}(t) \doteq \varphi_{n}\left(t, \alpha_{n}(t)\right)+\varphi_{n}^{*}\left(t, \beta_{n}(t)\right)-\left\langle\alpha_{n}(t), \beta_{n}(t)\right\rangle .
$$

Clearly, when $n \rightarrow \infty$, it converges a.e. to

$$
\theta(t) \doteq \varphi(t, \alpha(t))+\varphi^{*}(t, \beta(t))-\langle\alpha(t), \beta(t)\rangle
$$

and its integral, which is also its norm in $L^{1}(\Omega)$ converges to that of $\theta$. Applying Fatou's lemma to the positive sequence $\theta_{n}+\theta-\left|\theta_{n}-\theta\right|$ gives

$\liminf _{n \rightarrow \infty} \int_{\Omega}\left(\theta_{n}+\theta-\left|\theta_{n}-\theta\right|\right) \mathrm{d} \mu=2 \int_{\Omega} \theta \mathrm{d} \mu-\limsup _{n \rightarrow \infty} \int_{\Omega}\left|\theta_{n}-\theta\right| \mathrm{d} \mu \geq \int_{\Omega} 2 \theta \mathrm{d} \mu$,

which implies that $\theta_{n}$ converges to $\theta$ in $L^{1}(\Omega)$. Since $\left\langle\alpha_{n}(t), \beta_{n}(t)\right\rangle$ converges in the same space to $\langle\alpha, \beta\rangle$, this implies that $\varphi_{n}\left(t, \alpha_{n}(t)\right)+\varphi_{n}^{*}\left(t, \beta_{n}(t)\right)$ converges to $\varphi(t, \alpha(t))+\varphi^{*}(t, \beta(t))$ also in $L^{1}(\Omega)$. 
Proof of Theorem 3.19. We claim that (田) of Theorem 2.22 is satisfied for any $\mu>0$, e.g. $\mu=1$. First, for $\xi \in X$, inequality (3.4) gives here:

$$
\varphi_{n}(t, \xi)+\|\xi\|_{X}\left\|\beta_{n}(t)\right\|_{X^{\prime}}+\varphi_{n}^{*}\left(t, \beta_{n}(t)\right) \geq 0 .
$$

By Remark 3, it follows that

$$
\varphi_{n 1, p}(t, \xi)+\|\xi\|_{X}\left\|\beta_{n}(t)\right\|_{X^{\prime}}+\frac{1}{p}\left\|\beta_{n}(t)\right\|_{X^{\prime}}^{q}+\varphi^{*}\left(t, \beta_{n}(t)\right) \geq 0 .
$$

Replacing $\xi$ by $u(t)$ for arbitrary $u \in L^{p}(\Omega ; X)$ and integrating over $\Omega$ gives the two constants

$$
a_{n}=\|\beta\|_{L^{q}\left(\Omega ; X^{\prime}\right)} \text { and } b_{n}=\frac{1}{p}\left\|\beta_{n}\right\|_{L^{q}\left(\Omega ; X^{\prime}\right)}^{q}+\int_{\Omega} \varphi^{*}\left(t, \beta_{n}(t)\right) \mathrm{d} \mu .
$$

From the hypotheses, both sequences $a_{n}$ and $b_{n}$ are bounded, hence the two constants $a \doteq \sup _{n \in \mathbf{N}} a_{n}$ and $b \doteq \sup _{n \in \mathbf{N}} b_{n}$ are finite and satisfy the first condition of (用).

For $u \in L^{p}(\Omega ; X)$, and let $v_{n} \doteq J_{\lambda, p}^{\partial I_{\varphi_{n}}}(u)$. Corollary 3.9 implies that $v_{n}(t)=J_{\lambda, p}^{\partial \varphi_{n}(t)}(u(t))$ for a.e. $t$. By (ब) of Theorem 2.22 applied for almost every $t \in \Omega$, it follows that $v_{n}(t)$ converges to $v(t) \doteq J_{\lambda, p}^{\partial \varphi(t)}(u(t))$ for a.e. $t$. But inequality (3.5) applies here to give

$$
\begin{aligned}
\left\|v_{n}(t)\right\|_{X}^{p} \leq C_{p, \lambda}\left(\|u(t)\|_{X}^{p}+\left\|\alpha_{n}(t)\right\|_{X}^{p}\right. & \\
& \left.\left.+\left\|\beta_{n}(t)\right\|_{X^{\prime}}^{q}+\varphi_{n}\left(t, \alpha_{n}(t)\right)\right)+\varphi_{n}^{*}\left(t, \beta_{n}(t)\right)\right) .
\end{aligned}
$$

From the hypotheses of the theorem together with the last statement of Lemma 3.13, it follows that the right-hand side of (3.9) converges strongly in $L^{1}(\Omega)$. Consequently, by dominated convergence, $v_{n}$ converges strongly to $v$ in $L^{p}(\Omega ; X)$. This is the second condition of (田) in Theorem 2.22

Finally, the third condition of (Ifi) is satisfied by the sequence $\left\{\alpha_{n}\right\}$ itself. Indeed, by (1) of Theorem 2.22 applied for almost every $t \in \Omega$, it follows that $w_{n}(t) \doteq\left(\varphi_{n}\right)_{1, p}\left(t, \alpha_{n}(t)\right)$ converges to $w(t) \doteq \varphi(t, \alpha(t))$ for a.e. $t \in$ $\Omega$. Now applying Fatou's lemma to the sequence of non-negative functions $\varphi_{n}\left(t, \alpha_{n}(t)\right)-w_{n}(t)$ gives

$$
\begin{aligned}
\liminf _{n \rightarrow \infty} \int_{\Omega}\left(\varphi_{n}\left(t, \alpha_{n}(t)\right)-w_{n}(t)\right) \mathrm{d} \mu & =I_{\varphi}(\alpha)-\limsup _{n \rightarrow \infty} \int_{\Omega} w_{n}(t) \mathrm{d} \mu \\
& \geq \int_{\Omega}(\varphi(t, \alpha(t))-w(t)) \mathrm{d} \mu,
\end{aligned}
$$

from which it follows that $\lim \sup \int_{\Omega} w_{n}(t) \mathrm{d} \mu \leq \int_{\Omega} w(t) \mathrm{d} \mu$. A similar computation using the inequality (3.8) applied for $\xi=J_{\lambda, p}^{\partial \varphi_{n}(t)}\left(\alpha_{n}(t)\right)$ gives

$$
\liminf _{n \rightarrow \infty} \int_{\Omega} w_{n}(t) \mathrm{d} \mu \geq \int_{\Omega} w(t) \mathrm{d} \mu .
$$

In conclusion, $\int_{\Omega} w_{n}(t) \mathrm{d} \mu$ converges to $\int_{\Omega} w(t) \mathrm{d} \mu$, i.e. $\lim _{n \rightarrow \infty} I_{\varphi_{n}}\left(\alpha_{n}\right)=$ $I_{\varphi}(\alpha)$. 
Remark 12. The previous Theorem applies to the case where $\varphi_{n}(t) \stackrel{\mathcal{M}}{\longrightarrow} \varphi(t)$ for $t \in \Omega$ and there exists $m_{n} \in L^{1}(\Omega)$ and $\alpha>1$ such that $m_{n} \rightarrow m \in L^{1}(\Omega)$ and

$$
\alpha^{-1} \frac{\|\xi\|^{p}}{p}-m_{n}(t) \leq \varphi_{n}(t, \xi) \leq m_{n}(t)+\alpha \frac{\|\xi\|^{p}}{p} .
$$

Indeed one has then

$$
\alpha^{-1} \frac{\|\eta\|^{q}}{q}-m_{n}(t) \leq \varphi_{n}(t, \eta) \leq m_{n}(t)+\alpha \frac{\|\eta\|^{q}}{q},
$$

and it is thus clear that $\varphi_{n}\left(t, \alpha_{n}(t)\right), \varphi(t, \alpha(t)), \varphi_{n}^{*}\left(t, \beta_{n}(t)\right)$ and $\varphi^{*}(t, \beta(t))$ are all summable. By Lemma 2.17, one also has $\varphi_{n}(t, 0) \rightarrow \varphi(t, 0)$ and $\varphi_{n}^{*}(t, 0) \rightarrow \varphi^{*}(t, 0)$. By dominated convergence, both convergences $I_{\varphi_{n}}(0) \rightarrow$ $I_{\varphi}(0)$ and $I_{\varphi_{n}^{*}}(0) \rightarrow I_{\varphi^{*}}(0)$ follow. Therefore Theorem 3.12 applies.

\section{Periodic unfolding}

The periodic unfolding operator was introduced by Cioranescu, Damlamian and Griso [10]. We recall the definitions and properties of this operator. The proofs can be found in [10, 13, 20].

In $\mathbf{R}^{N}$, let $Y$ be a reference cell (e.g. $] 0,1\left[{ }^{N}\right.$, or more generally a set having the paving property with respect to a basis $\left(b_{1}, \ldots, b_{N}\right)$ defining the periods). For $y \in \mathbf{R}^{N},[y]_{Y}$ denotes the unique integer combination $\sum_{j=1}^{N} k_{j} b_{j}$, with $k_{j} \in \mathbf{Z}$, of the periods such that $y-[y]_{Y}$ belongs to $Y$ and define

$$
\{y\}_{Y}=y-[y]_{Y} \in Y .
$$

Definition 4.1. Let $Y$ be a reference cell, $\varepsilon$ a positive number, $S$ a set and a map $u: \mathbf{R}^{N} \rightarrow S$. The unfolding operator $\mathcal{T}_{\varepsilon}^{Y}$ is defined by

$$
\begin{aligned}
\mathcal{T}_{\varepsilon}^{Y}(u): \mathbf{R}^{N} \times \mathbf{R}^{N} & \rightarrow S \\
(x, y) & \mapsto \mathcal{T}_{\varepsilon}^{Y}(u)(x, y)=u\left(\varepsilon\left[\frac{x}{\varepsilon}\right]_{Y}+\varepsilon y\right) .
\end{aligned}
$$

One readily sees that for every $x \in \mathbf{R}^{N}$,

$$
\mathcal{T}_{\varepsilon}^{Y}(u)(x,\{x / \varepsilon\})=u(x) .
$$

Moreover, $\mathcal{T}_{\varepsilon}^{Y}(u)$ is invariant under the following action of $\mathbf{Z}^{N}$ : for $k \in \mathbf{Z}^{N}$,

$$
\mathcal{T}_{\varepsilon}^{Y}(u)(x+\varepsilon k, y-k)=\mathcal{T}_{\varepsilon}^{Y}(u)(x, y) .
$$

If $u: \mathbf{R}^{N} \rightarrow S$ and $f: S \rightarrow S^{\prime}$, then

$$
\mathcal{T}_{\varepsilon}^{Y}(f \circ u)=f \circ \mathcal{T}_{\varepsilon}^{Y}(u) .
$$


In particular if $u: \mathbf{R}^{N} \rightarrow S$ and $v: \mathbf{R}^{N} \rightarrow T$, the preceding property applied to the projections $P:(u, v) \mapsto u$ and $Q:(u, v) \mapsto v$ yields

$$
\mathcal{T}_{\varepsilon}^{Y}((u, v))=\left(\mathcal{T}_{\varepsilon}^{Y}(u), \mathcal{T}_{\varepsilon}^{Y}(v)\right) .
$$

Therefore, if $F: S \times T \rightarrow R$,

$$
\mathcal{T}_{\varepsilon}^{Y}(F(u, v))=F\left(\mathcal{T}_{\varepsilon}^{Y}(u), \mathcal{T}_{\varepsilon}^{Y}(v)\right) .
$$

Useful particular cases are when $S=\mathbf{R}, T=\mathbf{R}$ and $F:(s, t) \rightarrow s t$ and when $S=\mathbf{R}^{N}, T=\mathbf{R}^{N}$ and $F$ is the dot product.

Proposition 4.2. If $u \in L^{1}\left(\mathbf{R}^{N}\right)$, then $\mathcal{T}_{\varepsilon}^{Y}(u) \in L^{1}\left(\mathbf{R}^{n} \times Y\right)$ and

$$
\int_{\mathbf{R}^{N}} u(x) \mathrm{d} x=\frac{1}{|Y|} \int_{\mathbf{R}^{N} \times Y} \mathcal{T}_{\varepsilon}^{Y}(u)(x, y) \mathrm{d} x \mathrm{~d} y .
$$

In particular, if $1 \leq p<+\infty$ and $u \in L^{p}\left(\mathbf{R}^{N}\right)$, then $\mathcal{T}_{\varepsilon}^{Y}(u) \in L^{p}\left(\mathbf{R}^{N} \times Y\right)$, and

$$
\left\|\mathcal{T}_{\varepsilon}^{Y}(u)\right\|_{L^{p}\left(\mathbf{R}^{N} \times Y\right)}=|Y|^{1 / p}\|u\|_{L^{p}\left(\mathbf{R}^{N}\right)} .
$$

Remark 13. In the sequel, a function which is defined on a set $A$ of $\mathbf{R}^{N}$, can be viewed as a function defined on $\mathbf{R}^{N}$, if we consider its extension by 0 outside of $A$.

The characteristic function associated to the set $A$, is denoted by $\chi_{A}$. The combination of Proposition 4.2 together with (4.1) yields:

Proposition 4.3. Let $A \subset \mathbf{R}^{N}$ be measurable. If $u$ belongs to $L^{1}(A)$, then $\mathcal{T}_{\varepsilon}^{Y}\left(\chi_{A}\right) \mathcal{T}_{\varepsilon}^{Y}(u)$ is well-defined on $\mathbf{R}^{N} \times \mathbf{R}^{N}, \mathcal{T}_{\varepsilon}^{Y}\left(\chi_{A}\right) \mathcal{T}_{\varepsilon}^{Y}(u) \in L^{1}\left(\mathbf{R}^{N} \times Y\right)$, and

$$
\int_{A} u(x) \mathrm{d} x=\frac{1}{|Y|} \int_{\mathbf{R}^{N} \times Y} \mathcal{T}_{\varepsilon}^{Y}\left(\chi_{A}\right) \mathcal{T}_{\varepsilon}^{Y}(u) \mathrm{d} x \mathrm{~d} y .
$$

Moreover, if $1 \leq p<+\infty$ and $u \in L^{p}(A)$, then $\mathcal{T}_{\varepsilon}^{Y}\left(\chi_{A}\right) \mathcal{T}_{\varepsilon}^{Y}(u)$ is well-defined on $\mathbf{R}^{N} \times \mathbf{R}^{N}, \mathcal{T}_{\varepsilon}^{Y}\left(\chi_{A}\right) \mathcal{T}_{\varepsilon}^{Y}(u) \in L^{p}\left(\mathbf{R}^{N} \times Y\right)$ and

$$
\left\|\mathcal{T}_{\varepsilon}^{Y}\left(\chi_{A}\right) \mathcal{T}_{\varepsilon}^{Y}(u)\right\|_{L^{p}\left(\mathbf{R}^{N} \times Y\right)}=|Y|^{1 / p}\|u\|_{L^{p}(A)} .
$$

Since the unfolding operator has a local action, it is natural to examine its effect on locally summable functions.

Proposition 4.4. For every $1 \leq p<\infty, \mathcal{T}_{\varepsilon}^{Y}$ is a linear and continuous operator from $L_{\mathrm{loc}}^{p}\left(\mathbf{R}^{N}\right)$ to $L_{\mathrm{loc}}^{p}\left(\mathbf{R}^{N} \times \mathbf{R}^{N}\right)$.

We turn now to the $L_{\text {loc }}^{p}$ convergence properties for $1 \leq p<+\infty$.

Theorem 4.5. Let $\left(u_{\varepsilon}\right)_{\varepsilon}, u$ in $L_{\mathrm{loc}}^{p}\left(\mathbf{R}^{N}\right), 1 \leq p<+\infty$. If $u_{\varepsilon} \rightarrow u$ strongly in $L_{\mathrm{loc}}^{p}\left(\mathbf{R}^{N}\right)$ then

$$
\mathcal{T}_{\varepsilon}^{Y}\left(u_{\varepsilon}\right) \rightarrow u \otimes 1 \quad \text { strongly in } L_{\mathrm{loc}}^{p}\left(\mathbf{R}^{N} \times \mathbf{R}^{N}\right) \text { as } \varepsilon \rightarrow 0 .
$$


Global convergences follow easily.

Theorem 4.6. Let $A \subset \mathbf{R}^{N}$ be measurable $\left(u_{\varepsilon}\right)_{\varepsilon}, u$ in $L^{p}\left(\mathbf{R}^{N}\right), 1 \leq p<$ $+\infty$. If $u_{\varepsilon} \rightarrow u$ strongly in $L^{p}\left(\mathbf{R}^{N}\right)$, then

$$
\mathcal{T}_{\varepsilon}^{Y}\left(\chi_{A}\right) \mathcal{T}_{\varepsilon}^{Y}\left(u_{\varepsilon}\right) \rightarrow\left(\chi_{A} u\right) \otimes 1 \text { strongly in } L^{p}\left(\mathbf{R}^{N} \times Y\right) \text { as } \varepsilon \rightarrow 0,
$$

and

$$
\mathcal{T}_{\varepsilon}^{Y}\left(u_{\varepsilon}\right)_{\mid A \times Y} \rightarrow u \otimes 1 \text { strongly in } L^{p}(A \times Y) \text { as } \varepsilon \rightarrow 0 \text {. }
$$

The following result states that the limit (if it exists) of an unfolded sequence is periodic.

Lemma 4.7. Let $u_{\varepsilon} \in L_{\mathrm{loc}}^{1}\left(\mathbf{R}^{N}\right)\left(\operatorname{resp} L_{\mathrm{loc}}^{p}\left(\mathbf{R}^{N}\right)\right)$ and $\hat{u} \in L_{\mathrm{loc}}^{1}\left(\mathbf{R}^{N} \times \mathbf{R}^{N}\right)$ (resp $\left.L_{\mathrm{loc}}^{p}\left(\mathbf{R}^{N}\right)\right)$. If

$$
\left.\mathcal{T}_{\varepsilon}^{Y}\left(u_{\varepsilon}\right) \rightarrow \hat{u} * \text {-weakly in } \mathcal{M}\left(\mathbf{R}^{N} \times \mathbf{R}^{N}\right) \text {, (resp weakly in } L_{\mathrm{loc}}^{p}\left(\mathbf{R}^{N}\right)\right) \text {. }
$$

where $\mathcal{M}\left(\mathbf{R}^{N} \times \mathbf{R}^{N}\right)$ denotes the Radon measure space, then $\hat{u}$ is $Y$-periodic.

Next, we recall the properties of the unfolding operator applied on the gradient of some functions. If $u \in W_{\text {loc }}^{1, p}\left(\mathbf{R}^{N}\right)$ then by Proposition 4.4, $\mathcal{T}_{\varepsilon}^{Y}(u) \in L_{\text {loc }}^{p}\left(\mathbf{R}^{N} \times \mathbf{R}^{N}\right)$ and $\mathcal{T}_{\varepsilon}^{Y}(\nabla u) \in L_{\text {loc }}^{p}\left(\mathbf{R}^{N} \times \mathbf{R}^{N}\right)$. Moreover, for every test function $\varphi \in \mathcal{D}\left(\mathbf{R}^{N} \times \mathbf{R}^{N}\right)$

$$
\begin{array}{r}
\int_{\mathbf{R}^{N} \times \mathbf{R}^{N}} \nabla_{y} \varphi \mathcal{T}_{\varepsilon}^{Y}(u) \mathrm{d} x \mathrm{~d} y=\int_{\mathbf{R}^{N} \times \mathbf{R}^{N}} \nabla_{y} \varphi(x, y) u\left(\varepsilon[x / \varepsilon]_{Y}+\varepsilon y\right) \mathrm{d} x \mathrm{~d} y \\
=-\int_{\mathbf{R}^{N} \times \mathbf{R}^{N}} \varphi(x, y) \varepsilon \nabla u\left(\varepsilon[x / \varepsilon]_{Y}+\varepsilon y\right) \mathrm{d} x \mathrm{~d} y \\
=-\int_{\mathbf{R}^{N} \times \mathbf{R}^{N}} \varphi \varepsilon \mathcal{T}_{\varepsilon}^{Y}(\nabla u) \mathrm{d} x \mathrm{~d} y
\end{array}
$$

Therefore $\mathcal{T}_{\varepsilon}^{Y}(u)$ is weakly differentiable with respect to $y$, and

$$
\varepsilon \mathcal{T}_{\varepsilon}^{Y}(\nabla u)=\nabla_{y}\left(\mathcal{T}_{\varepsilon}^{Y}(u)\right) .
$$

The following result gives a relation between the limit of an unfolded sequence and the limit of the sequence:

Proposition 4.8. Let $\left(u_{\varepsilon}\right)_{\varepsilon}$ be a sequence of $L_{\mathrm{loc}}^{p}\left(\mathbf{R}^{N}\right)$ and let $u \in L_{\mathrm{loc}}^{p}\left(\mathbf{R}^{N}\right)$, $\hat{u} \in L_{\mathrm{loc}}^{p}\left(\mathbf{R}^{N} \times \mathbf{R}^{N}\right)$. Assume that $u_{\varepsilon} \rightarrow u$ weakly in $L_{\mathrm{loc}}^{p}\left(\mathbf{R}^{N}\right)$ and $\mathcal{T}_{\varepsilon}^{Y} u_{\varepsilon} \rightarrow \hat{u}$ weakly in $L_{\mathrm{loc}}^{p}\left(\mathbf{R}^{N} \times \mathbf{R}^{N}\right)$, then

$$
u(x)=\frac{1}{|Y|} \int_{Y} \hat{u}(x, y) \mathrm{d} y .
$$

The following proposition is an important tool for the sequel. 
Proposition 4.9. Let $\left(u_{\varepsilon}\right)_{\varepsilon}$ be a sequence in $W_{\text {loc }}^{1, p}\left(\mathbf{R}^{N}\right)$ and suppose that $\hat{u}$ belongs to $L_{\text {loc }}^{p}\left(\mathbf{R}^{N} ; \mathbf{R}^{N}\right)$. If $\left(u_{\varepsilon}\right)_{\varepsilon}$ is bounded in $L_{\text {loc }}^{p}\left(\mathbf{R}^{N}\right),\left(\varepsilon \nabla u_{\varepsilon}\right)_{\varepsilon}$ is bounded in $\left(L_{\mathrm{loc}}^{p}\left(\mathbf{R}^{N}\right)\right)^{N}$ and

$$
\mathcal{T}_{\varepsilon}^{Y}\left(u_{\varepsilon}\right) \rightarrow \hat{u} \text { weakly in } L_{\mathrm{loc}}^{p}\left(\mathbf{R}^{N} \times \mathbf{R}^{N}\right) \text { as } \varepsilon \rightarrow 0,
$$

then

$$
\varepsilon \mathcal{T}_{\varepsilon}^{Y}\left(\nabla u_{\varepsilon}\right) \rightarrow \nabla_{y} \hat{u} \text { weakly in } L_{\mathrm{loc}}^{p}\left(\mathbf{R}^{N} \times \mathbf{R}^{N}\right) \text { as } \varepsilon \rightarrow 0 .
$$

Moreover $\hat{u}$ is $Y$-periodic in $y$.

The following theorem is the main result.

Theorem 4.10. Let $\left(u_{\varepsilon}\right)_{\varepsilon}$ be a sequence which converges weakly to some $u$ in $W_{\text {loc }}^{1, p}\left(\mathbf{R}^{N}\right)$. Then, there exists a subsequence (not relabeled) and a function $\hat{u}$ in $L_{\mathrm{loc}}^{p}\left(\mathbf{R}^{N} ; W_{\text {loc }}^{1, p}\left(\mathbf{R}^{N}\right)\right)$ such that the following convergence holds:

$$
\mathcal{T}_{\varepsilon}^{Y}\left(\nabla u_{\varepsilon}\right) \rightarrow \nabla u \otimes 1+\nabla_{y} \hat{u},
$$

weakly in $L_{\mathrm{loc}}^{p}\left(\mathbf{R}^{N} \times \mathbf{R}^{N}\right)$ as $\varepsilon \rightarrow 0$. Additionally, $\hat{u}$ is $Y$-periodic.

\section{$5 \quad$ Homogenization results}

In this section we state the homogenization result, see [26] and [22]. We consider a problem of the form

$$
\left\{\begin{aligned}
(\nabla u, d) & \in \partial \varphi(x) & & \text { in } \Omega, \\
\operatorname{div} d & =-f & & \text { in } \Omega, \\
u & =0 & & \text { on } \partial \Omega,
\end{aligned}\right.
$$

where we assume

$$
\alpha^{-1} \frac{\|\xi\|^{p}}{p}-m(x) \leq \varphi(x, \xi) \leq \alpha \frac{\|\xi\|^{p}}{p}+m(x) .
$$

The variational formulation of (5.1), for given $f \in W^{-1, q}(\Omega)$, is

$$
\left\{\begin{array}{c}
\text { Find } u \in W_{0}^{1, p}(\Omega), \text { and } d \in L^{q}(\Omega)^{N} \text { such that } \\
d(x) \in \partial \varphi(x, \nabla u(x)) \quad \text { a.e. in } \Omega, \\
\int_{\Omega} d(x) \nabla \xi(x) \mathrm{d} x=\langle f, \xi\rangle, \forall \xi \in W_{0}^{1, p}(\Omega) .
\end{array}\right.
$$

This problem has at least one solution $(u, d) \in W_{0}^{1, p}(\Omega) \times L^{q}\left(\Omega ; \mathbf{R}^{N}\right)$. This is a consequence of more general results concerning the generalization to maximal monotone operators of the result of Leray and Lions 18] (17] or [8]), or from classical results of minimization of convex functionals together with the following characterization that is essentially a consequence of Proposition 2.12. 
Lemma 5.1. Let $\varphi$ be a lower semicontinuous function such that (5.2) holds. for $\alpha \geq 1$ and $m \in \mathrm{L}^{1}(\Omega)$. Then, for $f \in W^{-1, q}(\Omega)$, both

$$
\inf \left\{\int_{\Omega} \varphi(x, \nabla u(x)) \mathrm{d} x-\langle f, u\rangle: u \in W_{0}^{1, p}(\Omega)\right\}
$$

and

$$
\inf \left\{\int_{\Omega} \varphi^{*}(x, d(x)) \mathrm{d} x: \operatorname{div} d=-f\right\}
$$

are reached at $(u, d) \in W_{0}^{1, p}(\Omega) \times L^{q}(\Omega)$ if and only if $(u, d)$ solves (5.1). Therefore $(\nabla u, f) \in \partial I_{\varphi}$.

Proof. Define

$$
\begin{aligned}
\Phi: L^{p}\left(\Omega ; \mathbf{R}^{N}\right) & \rightarrow \mathbf{R} \\
e & \mapsto \int_{\Omega}(\varphi(x, e(x))-\langle d(x), e(x)\rangle) \mathrm{d} x
\end{aligned}
$$

and

$$
V=\left\{\nabla u: u \in W^{1, p}(\Omega)\right\} .
$$

By Proposition 3.6, one has

$$
\begin{aligned}
\Phi^{*}: L^{q}\left(\Omega ; \mathbf{R}^{N}\right) & \rightarrow \mathbf{R} \\
h & \mapsto \int_{\Omega} \varphi(x, h(x)-d(x)) \mathrm{d} x .
\end{aligned}
$$

and

$$
V^{\perp}=\left\{h \in L^{q}\left(\Omega, \mathbf{R}^{N}\right): \operatorname{div} h=0\right\} .
$$

Applying Proposition 2.12, one obtains

$$
\begin{aligned}
\int_{\Omega} \varphi(x, \nabla u(x)) \mathrm{d} x-\langle f, u\rangle & =\int_{\Omega}(\varphi(x, \nabla u(x))-\langle d(x), \nabla u(x)\rangle) \mathrm{d} x \\
& =\inf _{e \in V} \Phi(e)=-\inf _{h \in V^{\perp}} \Phi^{*}(h) \\
& =-\int_{\Omega} \varphi^{*}(x, d(x)) \mathrm{d} x .
\end{aligned}
$$

By Proposition 2.11, $(\nabla u(x), d(x)) \in \partial \varphi(x)$ for almost every $x \in \Omega$.

For the converse implication, note that by definition of subdifferentiability, for every $v \in W_{0}^{1, p}(\Omega)$

$$
\begin{aligned}
\int_{\Omega} \varphi(x, \nabla v(x)) \mathrm{d} x-\langle f, v\rangle & =\int_{\Omega} \varphi(x, \nabla v(x))-\langle d(x), \nabla v(x)\rangle \mathrm{d} x \\
& \geq \int_{\Omega} \varphi(x, \nabla u(x))-\langle d(x), \nabla u(x)\rangle \mathrm{d} x \\
& =\int_{\Omega} \varphi(x, \nabla u(x)) \mathrm{d} x-\langle f, u\rangle .
\end{aligned}
$$


Similarly, for every $h \in L^{q}\left(\Omega ; \mathbf{R}^{N}\right)$, with $\operatorname{div} h=-f$,

$$
\begin{aligned}
\int_{\Omega} \varphi^{*}(x, h(x)) \mathrm{d} x & \geq \int_{\Omega} \varphi^{*}(x, d(x))-\langle h(x)-d(x), \nabla u(x)\rangle \mathrm{d} x \\
& =\int_{\Omega} \varphi^{*}(x, d(x)) \mathrm{d} x
\end{aligned}
$$

since $\operatorname{div}(h-d)=0$.

Definition 5.2. Define

$$
\begin{aligned}
\Psi: W^{1, p}\left(\Omega ; \mathbf{R}^{N}\right) & \rightarrow \mathbf{R} \\
u & \mapsto \Psi(u)=I_{\varphi}(\nabla u)=\int_{\Omega} \varphi(x, \nabla u(x)) \mathrm{d} x,
\end{aligned}
$$

and

$$
\begin{aligned}
\tilde{\Psi}: L^{p}\left(\Omega ; \mathbf{R}^{N}\right) & \rightarrow \mathbf{R} \\
u & \mapsto\left\{\begin{aligned}
\Psi(u) & \text { if } u \in W^{1, p}\left(\Omega ; \mathbf{R}^{N}\right), \\
+\infty & \text { if } u \in L^{p}\left(\Omega ; \mathbf{R}^{N}\right) \backslash W^{1, p}\left(\Omega ; \mathbf{R}^{N}\right) .
\end{aligned}\right.
\end{aligned}
$$

Corollary 5.3. Let $f \in W^{-1, q}\left(\Omega ; \mathbf{R}^{N}\right)$ and $u \in W_{0}^{1, p}\left(\Omega ; \mathbf{R}^{N}\right)$, then $(u, f) \in$ $\partial \Psi$ iff there exists $d \in L^{q}\left(\Omega ; \mathbf{R}^{N}\right)$ such that $(u, d)$ solves (5.1).

Let $f \in L^{q}\left(\Omega ; \mathbf{R}^{N}\right)$ and $u \in W_{0}^{1, p}\left(\Omega ; \mathbf{R}^{N}\right)$, then $(u, f) \in \partial \tilde{\Psi}$ iff there exists $d \in L^{q}\left(\Omega ; \mathbf{R}^{N}\right)$ such that $(u, d)$ solves (5.1).

In both cases, $\Psi^{*}(f)=I_{\varphi^{*}}(d)$ (resp. $\left.\tilde{\Psi}^{*}(f)=I_{\varphi^{*}}(d)\right)\left(I_{\varphi^{*}}(d)\right.$ is unique even if $d$ is not).

Theorem 5.4. Let $1<p<\infty, p^{-1}+q^{-1}=1, m_{\varepsilon} \in L^{1}(\Omega), \alpha \in(0,1]$, $\Omega \subset \mathbf{R}^{N}$ and let $\varphi_{\varepsilon}: \Omega \times \mathbf{R}^{N} \rightarrow \mathbf{R}$. Assume that $\varphi(x, \cdot)$ is convex for every $x \in \Omega, \varphi(\cdot, \xi)$ is measurable for every $\xi \in \mathbf{R}^{N}$ and for almost every $x \in \Omega$ and for every $\xi \in \mathbf{R}^{N}$,

$$
\alpha \frac{\|\xi\|^{p}}{p}-m_{\varepsilon}(x) \leq \varphi_{\varepsilon}(x, \xi) \leq m_{\varepsilon}(x)+\alpha^{-1} \frac{\|\xi\|^{p}}{p} .
$$

Suppose that there exists a cell $Y \subset \mathbf{R}^{N}$ and a function $\varphi: \Omega \times Y \times \mathbf{R}^{N} \rightarrow \mathbf{R}$ and $m \in L^{1}(\Omega \times Y)$ such that for almost every $(x, y) \in \Omega \times Y$,

$$
\mathcal{T}_{\varepsilon}^{Y} \varphi_{\varepsilon}(x, y, .) \rightarrow \varphi(x, y, .),
$$

and $\mathcal{T}_{\varepsilon}\left(m_{\varepsilon}\right) \rightarrow m$ strongly in $L^{1}(\Omega \times Y)$ as $\varepsilon \rightarrow 0$.

Finally, assume that $f_{\varepsilon} \rightarrow f_{0}$ strongly in $W^{-1, q}(\Omega)$ as $\varepsilon \rightarrow 0$.

Consider a (not necessarily unique) solution $\left(u_{\varepsilon}, d_{\varepsilon}\right) \in W_{0}^{1, p}(\Omega) \times L^{q}\left(\Omega, \mathbf{R}^{n}\right)$ of the problem

$$
\left\{\begin{aligned}
\left(\nabla u_{\varepsilon}, d_{\varepsilon}\right) & \in \partial \varphi_{\varepsilon}(x) & & \text { in } \Omega, \\
-\operatorname{div} d_{\varepsilon} & =f_{\varepsilon} & & \text { in } \Omega, \\
u_{\varepsilon} & =0 & & \text { on } \partial \Omega .
\end{aligned}\right.
$$


Then, the family $\left(u_{\varepsilon}, d_{\varepsilon}\right)_{\varepsilon>0}$ is weakly compact in $W_{0}^{1, p}(\Omega) \times L^{q}\left(\Omega ; \mathbf{R}^{N}\right)$. Moreover, if $u_{0}$ is any of its weak limit points, i.e. if there is $\left(\varepsilon_{n}\right)_{n \geq 1}$ such that $\varepsilon_{n} \rightarrow 0$

$$
\begin{array}{ll}
u_{\varepsilon_{n}} \rightarrow u_{0} & \text { weakly in } W_{0}^{1, p}(\Omega), \\
d_{\varepsilon_{n}} \rightarrow d_{0} & \text { weakly in } L^{q}\left(\Omega ; \mathbf{R}^{N}\right),
\end{array}
$$

then

$$
\left\{\begin{aligned}
\left(\nabla u_{0}, d_{0}\right) & \in \partial \varphi_{0}(x) & & \text { in } \Omega, \\
-\operatorname{div} d_{0} & =f_{0} & & \text { in } \Omega, \\
u_{0} & =0 & & \text { on } \partial \Omega,
\end{aligned}\right.
$$

where, for almost every $x \in \Omega$, the function $\varphi_{0}$ is given by

$$
\varphi_{0}(x, \xi)=\inf \left\{\frac{1}{|Y|} \int_{Y} \varphi\left(x, y, \xi+\nabla_{y} \hat{u}(y)\right) \mathrm{d} y: \hat{u} \in W_{\text {per }}^{1, p}(Y)\right\} .
$$

The function $\varphi_{0}$ is lower semicontinuous convex in the second argument and satisfies (5.4), with

$$
\bar{m}(x)=\frac{1}{|Y|} \int_{Y} m(x, y) d y
$$

And as $n \rightarrow+\infty$,

$$
\begin{gathered}
\Psi_{\varepsilon_{n}}\left(u_{\varepsilon_{n}}\right)=\int_{\Omega} \varphi_{\varepsilon_{n}}\left(x, \nabla u_{\varepsilon_{n}}(x)\right) \mathrm{d} x \rightarrow \int_{\Omega} \varphi_{0}\left(x, \nabla u_{0}(x)\right) \mathrm{d} x=\Psi_{0}\left(u_{0}\right), \\
\Psi_{\varepsilon_{n}}^{*}\left(f_{\varepsilon_{n}}\right)=\int_{\Omega} \varphi_{\varepsilon_{n}}^{*}\left(x, d_{\varepsilon_{n}}(x)\right) \mathrm{d} x \rightarrow \int_{\Omega} \varphi_{0}^{*}\left(x, d_{0}(x)\right) \mathrm{d} x=\Psi_{0}^{*}\left(f_{0}\right) .
\end{gathered}
$$

Remark 14. Theorem 5.4 applies to the particular case where $\varphi_{\varepsilon}(x)=\Phi\left(\frac{x}{\varepsilon}\right)$ and $m_{\varepsilon}(x)=M\left(\frac{x}{\varepsilon}\right)$ where $\varphi(x, y, \cdot)=\Phi(y, \cdot)$ and $m(x, y)=M(y)$. In the most general situation, given any $\varphi(x, y)$ satisfying the corresponding condition (5.4), one can construct a sequence $\varphi_{\varepsilon}(x, \cdot)$ to obtain $\mathcal{T}_{\varepsilon}^{Y} \varphi_{\varepsilon}(x, y,.) \rightarrow$ $\varphi(x, y,).(13])$.

Proof of Theorem 5.4. First note that by (5.4), for every $\eta \in \mathbf{R}^{N}$,

$$
\alpha^{-1} \frac{\|\eta\|^{q}}{q}-m_{\varepsilon}(x) \leq \varphi_{\varepsilon}^{*}(x, \eta) \leq m_{\varepsilon}(x)+\alpha \frac{\|\eta\|^{p}}{p} .
$$

By Proposition 2.11,

$$
\alpha^{-1}\left(\frac{\left\|\nabla u_{\varepsilon}\right\|_{q}^{q}}{q}+\frac{\left\|d_{\varepsilon}\right\|_{q}^{q}}{q}\right) \leq \int_{\Omega} \varphi_{\varepsilon}\left(x, \nabla u_{\varepsilon}(x)\right)+\varphi_{\varepsilon}^{*}\left(x, d_{\varepsilon}(x)\right) \mathrm{d} x=\left\langle f_{\varepsilon}, u_{\varepsilon}\right\rangle .
$$

Since $p>1$, the sequences $\left(u_{\varepsilon}\right)_{\varepsilon>0}$ and $\left(d_{\varepsilon}\right)_{\varepsilon>0}$ are thus bounded in $W^{1, p}(\Omega)$ and $L^{q}(\Omega)$. Hence they are weakly compact. 
Assume now that $u_{\varepsilon_{n}} \rightarrow u_{0}$ weakly in $W_{0}^{1, p}(\Omega)$ and $d_{\varepsilon_{n}} \rightarrow d_{0}$ weakly in $L^{q}\left(\mathbf{R}^{N} ; \mathbf{R}^{N}\right)$. By Theorem 4.10 , there is $\hat{u} \in L^{p}\left(\Omega ; W_{\text {per }}^{1, p}(\Omega)\right)$ such that, still up to subsequences

$$
\mathcal{T}_{\varepsilon_{n}}^{Y}\left(\nabla u_{\varepsilon_{n}}\right) \rightarrow \nabla_{x} u_{0}+\nabla_{y} \hat{u} \text { weakly in } L^{p}\left(\mathbf{R}^{N} \times Y ; \mathbf{R}^{N}\right) .
$$

Moreover, the sequence $\left(\mathcal{T}_{\varepsilon_{n}}^{Y}\left(d_{\varepsilon_{n}}\right)\right)_{n>1}$ is bounded in $L^{q}\left(\mathbf{R}^{N} \times Y\right)$; hence there exists $\eta \in L^{q}\left(\mathbf{R}^{N} \times Y\right)$, so that up to a subsequence, $\mathcal{T}_{\varepsilon_{n}}^{Y}\left(d_{\varepsilon_{n}}\right) \rightarrow \eta$. Letting $\hat{d}=\eta-\frac{1}{|Y|} \int_{Y} \eta(y) \mathrm{d} y$, one has

$$
\mathcal{T}_{\varepsilon_{n}}^{Y}\left(d_{\varepsilon_{n}}\right) \rightarrow d_{0}+\hat{d} \text { weakly in } L^{q}\left(\mathbf{R}^{N} \times Y\right) .
$$

Let $v \in \mathcal{C}_{c}^{\infty}(\Omega)$. By the weak convergence of $\left(d_{\varepsilon_{n}}\right)_{n \geq 1}$ and the strong convergence of $\left(f_{\varepsilon_{n}}\right)_{n \geq 1}$, letting $n \rightarrow \infty$ in (5.6) yields

$$
\int_{\Omega}\left\langle d_{0}(x), \nabla v(x)\right\rangle \mathrm{d} x=\int_{\Omega} f_{0} v \mathrm{~d} x,
$$

i.e.

$$
-\operatorname{div} d_{0}=f_{0} \text { in } \mathcal{D}^{\prime}(\Omega) .
$$

Next, let $v \in \mathcal{D}(\Omega)$ and $w \in C^{\infty}\left(\mathbf{R}^{N}\right)$, such that $w$ is $Y$-periodic. Defining $v_{\varepsilon_{n}}(x)=\varepsilon_{n} v(x) w\left(x / \varepsilon_{n}\right)$, one has, by (5.6) and Proposition 4.3.

$$
\frac{1}{|Y|} \int_{\mathbf{R}^{N} \times Y}\left\langle\mathcal{T}_{\varepsilon_{n}}\left(d_{\varepsilon_{n}}\right), \mathcal{T}_{\varepsilon_{n}}^{Y}\left(\nabla v_{\varepsilon_{n}}\right)\right\rangle \mathrm{d} x \mathrm{~d} y=\int_{\Omega} f_{\varepsilon_{n}} v_{\varepsilon_{n}} \mathrm{~d} x .
$$

Letting $\varepsilon_{n} \rightarrow 0$, one has $v_{\varepsilon_{n}} \rightarrow 0$ weakly in $W^{1, p}(\Omega)$ and $\mathcal{T}_{\varepsilon_{n}}^{Y}\left(\nabla v_{\varepsilon_{n}}\right) \rightarrow$ $v(x) \nabla_{y} w(y)$ strongly in $L^{p}\left(\Omega ; W_{\text {per }}^{1, p}(Y)\right)$, so that

$$
\frac{1}{|Y|} \int_{\Omega \times Y}\left\langle d_{0}(x)+\hat{d}(x, y), v(x) \nabla_{y} w(y)\right\rangle \mathrm{d} x \mathrm{~d} y=0 .
$$

Since $v$ is arbitrary, one concludes that for almost every $x \in \Omega$, for every $w \in W_{\text {per }}^{1, p}(Y)$,

$$
\frac{1}{|Y|} \int_{Y}\left\langle\hat{d}(x, y), \nabla_{y} w(y)\right\rangle \mathrm{d} y=0,
$$

i.e. $-\operatorname{div} \hat{d}(x, \cdot)=0$ in $\left(\mathcal{C}_{\text {per }}^{\infty}\right)^{\prime}(Y)$.

By the Mosco convergence one obtains

$$
\begin{aligned}
& \int_{\Omega \times Y} \varphi\left(x, \nabla u_{0}(x)+\nabla_{y} \hat{u}(x, y)\right)+\varphi^{*}\left(x, d_{0}(x)+\hat{d}(x, y)\right) \mathrm{d} x \\
& \leq \liminf _{n \rightarrow \infty} \int_{\Omega \times Y} \mathcal{T}_{\varepsilon_{n}} \varphi_{\varepsilon_{n}}\left(x, \nabla \mathcal{T}_{\varepsilon_{n}} u_{\varepsilon_{n}}(x)\right)+\mathcal{T}_{\varepsilon_{n}} \varphi_{\varepsilon_{n}}^{*}\left(x, \mathcal{T}_{\varepsilon_{n}} d_{\varepsilon}(x)\right) \mathrm{d} x \\
&=\liminf _{n \rightarrow \infty}\left\langle f_{\varepsilon_{n}}, u_{\varepsilon_{n}}\right\rangle .
\end{aligned}
$$


By assumption, one has

$$
\left\langle f_{\varepsilon}, u_{\varepsilon}\right\rangle \rightarrow\left\langle f_{0}, u_{0}\right\rangle=\int_{\Omega}\left\langle d_{0}(x), \nabla u_{0}(x)\right\rangle \mathrm{d} x .
$$

By Proposition 2.11, one concludes that

$$
\begin{aligned}
\int_{\Omega \times Y} \varphi\left(x, \nabla u_{0}(x)+\nabla_{y} \hat{u}(x, y)\right)+\varphi^{*}\left(x, d_{0}(x)\right. & +\hat{d}(x, y)) \mathrm{d} x \\
& =\int_{\Omega}\left\langle d_{0}(x), \nabla u_{0}(x)\right\rangle \mathrm{d} x,
\end{aligned}
$$

therefore, in view of Lemma 5.5 below, $\left(u_{0}(x), d_{0}(x)\right) \in \partial \varphi_{0}(x)$. Finally, one has that

$$
\begin{aligned}
& \liminf _{n \rightarrow \infty} \int_{\Omega \times Y} \mathcal{T}_{\varepsilon_{n}} \varphi_{\varepsilon_{n}}\left(x, \nabla \mathcal{T}_{\varepsilon_{n}} u_{\varepsilon_{n}}(x)\right) \mathrm{d} x \mathrm{~d} y \\
&=\int_{\Omega \times Y} \varphi\left(x, \nabla u_{0}(x)+\nabla_{y} \hat{u}(x, y)\right) \mathrm{d} x \mathrm{~d} y,
\end{aligned}
$$

and

$$
\begin{aligned}
\liminf _{n \rightarrow \infty} \int_{\Omega \times Y} \mathcal{T}_{\varepsilon_{n}} \varphi_{\varepsilon_{n}}^{*}\left(x, \nabla \mathcal{T}_{\varepsilon_{n}} u_{\varepsilon_{n}}(x)\right) \mathrm{d} x \mathrm{~d} y & \\
= & \int_{\Omega \times Y} \varphi^{*}\left(x, d_{0}(x)+\hat{d}(x, y)\right) \mathrm{d} x \mathrm{~d} y .
\end{aligned}
$$

Since, by Lemma 5.1, the right-hand sides are independent of the solution of problem 5.7 and since the reasoning can be made for every subsequence, one has the required convergences of the integrals.

Remark 15 . The previous proof easily extends to different boundary conditions for which a variational formulation holds. It can be extended to the reiterated case as in [20].

The properties of the homogenized function are given now.

Lemma 5.5. If $\varphi: Y \times \mathbf{R}^{N} \rightarrow \mathbf{R}$ satisfies

$$
\alpha^{-1} \frac{\|\xi\|^{p}}{p}-m(y) \leq \varphi(y, \xi) \leq \alpha \frac{\|\xi\|^{p}}{p}+m(y),
$$

then $\psi: \mathbf{R}^{N} \rightarrow \mathbf{R}$ defined by

$$
\psi(\xi)=\inf \left\{\frac{1}{|Y|} \int_{Y} \varphi\left(y, \xi+\nabla_{y} \hat{u}(y)\right) \mathrm{d} y: \hat{u} \in W_{\text {per }}^{1, p}(Y)\right\},
$$

satisfies (5.15) with

$$
\bar{m}(x)=\frac{1}{|Y|} \int_{Y} m(x, y) d y
$$


and

$$
\begin{aligned}
\psi^{*}(\eta)=\inf \left\{\frac{1}{|Y|} \int_{Y} \varphi^{*}(\eta+\hat{d}(y)) \mathrm{d} y\right. & : \hat{d} \in L^{q}(Y), \\
& \left.\frac{1}{|Y|} \int_{Y} \hat{d} \mathrm{~d} y=0 \text { and } \operatorname{div} \hat{d}=0\right\}
\end{aligned}
$$

Proof. First note that

$$
\psi(\xi) \leq \frac{1}{|Y|} \int_{Y} \varphi(y, \xi) \mathrm{d} y \leq \frac{1}{|Y|} \int_{Y} m(y) \mathrm{d} y+\alpha \frac{\|\xi\|_{p}^{p}}{p} .
$$

By Jensen's inequality, one also has

$$
\alpha^{-1} \frac{\|\xi\|_{p}^{p}}{p}-\bar{m} \leq \int_{Y}\left(\alpha^{-1} \frac{\|\xi+\nabla \hat{u}(y)\|_{p}^{p}}{p}-m(y)\right) \mathrm{d} y \leq \int_{Y} \alpha^{-1} \mathrm{~d} y,
$$

so that

$$
\alpha^{-1} \frac{\|\xi\|_{p}^{p}}{p}-\bar{m} \leq \psi(\xi)
$$

and $\psi$ satisfies the required coercivity and growth conditions.

Consider now the convex functional

$$
\begin{aligned}
\Phi_{\xi}: L^{p}(Y) & \rightarrow \mathbf{R} \\
e & \mapsto \frac{1}{|Y|} \int_{Y} \varphi(y, \xi+e(y)) \mathrm{d} y
\end{aligned}
$$

and the vector space

$$
V=\left\{\nabla \hat{u}: \hat{u} \in W_{\text {per }}^{1, p}(Y)\right\}
$$

One has

$$
\Phi_{\xi}^{*}(d)=\frac{1}{|Y|} \int_{Y} \varphi(y, d(y))-\langle d(y), \xi\rangle \mathrm{d} y
$$

and

$$
V^{\perp}=\left\{\hat{d} \in L^{q}(Y): \frac{1}{|Y|} \int_{Y} \hat{d} \mathrm{~d} y=0 \text { and } \operatorname{div} \hat{d}=0\right\} .
$$

Applying Proposition 2.12, one obtains (5.16). Reiterating a similar argument, one finally obtains $\psi^{* *}=\psi$, so that $\psi$ is lower semicontinuous and convex.

Corollary 5.6. Under assumptions of Theorem 5.4, one has the following Mosco convergence:

$$
\tilde{\Psi}_{\varepsilon} \stackrel{\mathcal{M}}{\longrightarrow} \tilde{\Psi}_{0}
$$


Proof. We show that (何) of Theorem 2.22 is satisfied. The lower bound is easily obtained from (5.4) and Remark 5.4.

Let now $w$ be given in $L^{p}(\Omega)$. By Corollary 5.3, the value of the resolvant $J_{1, p}^{\partial \tilde{\Psi}_{\varepsilon}}(w) \doteq u_{\varepsilon}$ is the solution of the following variational problem (see (5.3)):

$$
\left\{\begin{array}{c}
\text { Find } u_{\varepsilon} \in W_{0}^{1, p}(\Omega), \text { and } d_{\varepsilon} \in L^{q}(\Omega)^{N} \text { such that } \\
d_{\varepsilon}(x) \in \partial \varphi_{\varepsilon}\left(x, \nabla u_{\varepsilon}(x)\right) \quad \text { a.e. in } \Omega \\
\int_{\Omega} d_{\varepsilon}(x) \cdot \nabla \xi(x) \mathrm{d} x=\left\langle v_{\varepsilon}, \xi\right\rangle \forall \xi \in W_{0}^{1, p}(\Omega), \\
\text { where } v_{\varepsilon}=F_{p}\left(w-u_{\varepsilon}\right)=\left|w-u_{\varepsilon}\right|^{p-2}\left(w-u_{\varepsilon}\right) .
\end{array}\right.
$$

It is then easy to check that $u_{\varepsilon}$ is bounded in $W_{0}^{1, p}(\Omega)$, and that $d_{\varepsilon}$ is bounded in $L^{q}(\Omega)^{N}$. Consider a sequence $\left\{\varepsilon_{n}\right\}$ such that $u_{\varepsilon_{n}}$ converge weakly to some $u_{0} \in W_{0}^{1, p}(\Omega)$ and $d_{\varepsilon_{n}}$ converges weakly to some $d_{0}$ in $L^{q}(\Omega)^{N}$. By compact embedding, $u_{\varepsilon_{n}}$ converges strongly to $u_{0}$ in $L^{p}(\Omega)$, so that $v_{\varepsilon_{n}}$ converges strongly to $v_{0} \doteq F_{p}\left(u-u_{0}\right)$. By Theorem 5.4, it follows that $u_{0}$ is the solution of

$$
\left\{\begin{array}{c}
\text { Find } u_{0} \in W_{0}^{1, p}(\Omega), \text { and } d_{0} \in L^{q}(\Omega)^{N} \text { such that } \\
d_{0}(x) \in \partial \varphi_{0}\left(x, \nabla u_{0}(x)\right) \quad \text { a.e. in } \Omega \\
\int_{\Omega} d_{0}(x) \cdot \nabla \xi(x) \mathrm{d} x=\left\langle v_{0}, \xi\right\rangle \forall \xi \in W_{0}^{1, p}(\Omega), \\
\text { where } v_{0}=F_{p}\left(w-u_{0}\right)=\left|w-u_{0}\right|^{p-2}\left(w-u_{0}\right) .
\end{array}\right.
$$

This just means that $u_{0}$ is $J_{1, p}^{\partial \tilde{\Psi}_{0}}(w)$, is therefore unique and is the strong limit of the whole sequence $J_{1, p}^{\partial \tilde{\Psi}_{\varepsilon}}(w)$ in $L^{p}(\Omega)$. This proves the second condition of (佃) of Theorem 2.22 .

Theorem 5.4 also implies that $\tilde{\Psi}_{\varepsilon}\left(u_{\varepsilon}\right)$ converges to $\tilde{\Psi}_{0}\left(u_{0}\right)$. On the other hand, $\left(\tilde{\Psi}_{\varepsilon}\right)_{1, p}(w)=\tilde{\Psi}_{\varepsilon}\left(u_{\varepsilon}\right)+\frac{1}{p}\left\|w-u_{\varepsilon}\right\|_{L^{p}(\Omega)}^{p}$ so that it converges to $\tilde{\Psi}_{0}\left(u_{0}\right)+\frac{1}{p}\left\|w-u_{0}\right\|_{L^{p}(\Omega)}^{p}$, which is exactly $\left(\tilde{\Psi}_{0}\right)_{1, p}(w)$. This satisfies the last condition of (迪) of Theorem 2.22 .

The same argument, making use of the uniform coerciveness of the $\Psi_{\varepsilon}$ on $W^{1, p}(\Omega)$ due to condition (5.4), shows the following convergence:

Corollary 5.7. The following weak $\Gamma$ convergence holds in $W^{1, p}(\Omega)$ :

$$
\Psi_{\varepsilon} \stackrel{w-\Gamma}{\longrightarrow} \Psi_{0} .
$$

\section{Acknowledgement}

The third author (JVS) was supported by the Fonds de la Recherche Scientifique-FNRS (Communauté française de Belgique), and the Fonds spécial de recherche (Université catholique de Louvain). 


\section{References}

[1] H. Atтouch, Famille d'opérateurs maximaux monotones et mesurabilité, Annali di Matematica pura et applicata, IV, Vol CXX, (1979), 35-111.

[2] H. Atrouch, Variational convergence for functions and operators, Applicable Mathematics Series, Pitman, Boston, Mass.-London, 1984.

[3] D. AzÉ, Caractérisation de la convergence au sens de Mosco en terme d'approximations inf-convolutives, Ann. faculté des sciences de Toulouse, Sér. 5, 8 No. 3 (1986-1987), 293-314.

[4] H. Brezis, Opérateurs maximaux monotones et semi-groupes de contractions dans les espaces de Hilbert, North Holland Mathematics Studies, No. 5. Notas de Mathematica (50). North Holland Publishing Co., Amsterdam-London; American Elsevier Publishing Co. Inc., New York, 1973, vi+183pp.

[5] F.E. Browder, Nonlinear variational inequalities and maximal monotone mappings in Banach spaces, Math. Ann. 183 (1969), 213-231.

[6] F.E. Browder, Nonlinear operators and nonlinear equations of evolution in Banach spaces, Nonlinear functional Analysis, Proc. Sympos. Pure Math. 18 Ameri. Math. Soc., 1976.

[7] C. Castaing and M. Valadier, Convex analysis and measurable multifunctions, Lecture Notes in Mathematics Studies, Vol. 580, Springer Verlag, 1977.

[8] V. Chiado'Piat, G. Dal Maso, and A. Defranceschi, G-convergence of monotone operators and homogenization, C. Ann. Inst. H. Poincaré Anal. Non Linéaire 7 (3) (1990), 123-160.

[9] V. Chiado'Piat and G. Dal Maso, Homogenization of monotone operators, Nonlinear Anal. 14 (9) (1990), 717-732.

[10] D. Cioranescu, A. Damlamian, and G. Griso, Periodic unfolding and homogenization, C. R. Math. Acad. Sci. Paris 335 (2002), no. 1, 99-104.

[11] G. Dal Maso, An introduction to $\Gamma$-convergence, Progress in linear diff. eq. and their app., Birkhauser, Boston, 1993.

[12] A. Damlamian, Convergence et dualité des fonctions convexes dans les espaces de Banach reflexifs, unpublished, 1975. 
[13] A. Damlamian, An elementary introduction to Periodic Unfolding, Multiscale problems and Asymptotic Analysis ; proceedings of the Midnight Sun Narvik Conference, June 2004, eds, GAKUTO International Series, Math.Sci. Appl. Vol. 24 (2005), Tokyo, p. 119-136, editors D. Lukkassen, A. Meidell and A. Piatnitski.

[14] A. Damlamian, N. Meunier and J. Van Schaftingen, Periodic homogenization of monotone multivalued operators, Nonlinear Anal., 67 (2007), no. 12, 3217-3239.

[15] E. De Giorgi and T. Franzoni, Su un tipo di convergenza variazionale, Atti Accad. Naz. Lincei Rend. Cl. Sci. Fis. Mat. Natur. 8, 58 (1975), no. 6, 842-850.

[16] I. Ekeland, and R. Temam, Convex analysis and variational problems, North Holland, 1972.

[17] G. Francfort, F. Murat and L. Tartar Monotone operators in divergence form with $x$-dependent multivalued graphs, Boll. U. M. I. 8 (2004), 23-59.

[18] J. LeRAY and J. L. Lions Quelques résultats de Visik sur les problemes elliptiques nonlinéaires par les méthodes de Minty-Browder, Bull. Soc. Math. France, 93 (1965), 97-107.

[19] N. Meunier and J. Van Schaftingen, Reiterated homogenization for elliptic operators, C. R. Acad. Sci. Paris, 340 (2005), no. 3, 209-214.

[20] N. Meunier and J. Van Schaftingen, Reiterated homogenization for elliptic functions, J. Math. Pures Appl. 84 (2005), 1716-1743.

[21] U. Mosco, Convergence of convex sets and of solutions of variational inequalities, Advances Math. 3 (1969), 510-585.

[22] F. Murat and L. Tartar H-convergence, Topics in the Mathematical Modelling of Composite Materials (1997), 21-43.

[23] R. T. RockAfEllaR Characterization of the subdifferentials of convex functions, Pacific J. Math. 17 (1966), 497-510.

[24] R. T. Rockafellar Integrals which are convex functionals. Pacific J. Math. 24, (1968), 525-539.

[25] R. T. RockAfEllar On the maximal monotonicity of subdifferential mappings, Pacific J. Math. 33 (1970), 209-216.

[26] L. Tartar, Cours Peccot, Collège de France, (1977). 
[27] S. L. Troyanski, On locally uniformly convex and differentiable norms in certain non-separable Banach spaces, Studia Math. 37 (1970/71), 173180. 